\title{
THE FREE BOUNDARY FOR ELASTIC-PLASTIC TORSION PROBLEMS ${ }^{1}$

\author{
BY
}

\author{
LUIS A. CAFFARELLI AND AVNER FRIEDMAN
}

\begin{abstract}
Consider the variational inequality: find $u \in K$ such that $\int_{Q} \nabla u$ - $\nabla(v-u)>\mu \int_{Q}(v-u)(\mu>0)$ for any $v \in K$, where $K=\{w \in$ $\left.H_{0}^{1}(Q),|\nabla w|<1\right\}$ and $Q$ is a simply connected domain whose boundary is piecewise $C^{3}$. The solution $u$ represents the stress function in a torsion problem of an elastic bar with cross section $Q$; the sets $E=\{x \in Q$; $|\nabla u(x)|<1\}, P=\{x \in Q ;|\nabla u(x)|=1\}$ are the elastic and plastic subsets of $Q$. The ridge $R$ of $Q$ is, by definition, the set of points in $Q$ where $\operatorname{dist}(x, \partial Q)$ is not $C^{1,1}$. The paper studies the location and shape of $E, P$ and the free boundary $\Gamma=\partial E \cap Q$. It is proved that the ridge is elastic and that $E$ is contained in a $(c / \mu)$-neighborhood of $R$, as $\mu \rightarrow \infty(c>0)$. The behavior of $E$ and $P$ near the vertices of $\partial Q$ is studied in detail, as well as the nature of $\Gamma$ away from the vertices. Applications are given to special domains. The case where $Q$ is multiply connected is also studied; in this case the definition of $K$ is somewhat different. Some results on the "upper plasticity" and "lower plasticity" and on the behavior as $\mu \rightarrow \infty$ are obtained.
\end{abstract}

1. Introduction. Let $Q$ be a 2-dimensional, simply connected bounded domain whose boundary $\partial Q$ consists of a finite number of $C^{3}$ closed arcs $S_{1}, \ldots, S_{m}$. We assume that these arcs are mutually disjoint except that the terminal end-point of each $S_{i}$ coincides with the initial end-point of $S_{i+1}$ (we agree to set $S_{m+1}=S_{1}$ ); this common point is denoted by $V_{i}$ and is called a vertex of $Q$.

Let

$$
K=\left\{v \in H_{0}^{1}(Q),|\nabla v| \leqslant 1\right\} .
$$

Consider the variational inequality

$$
\iint_{Q} \nabla u \cdot \nabla(v-u) \geqslant \mu \iint_{Q}(v-u) \text { for any } v \in K ; u \in K,
$$

where $\mu$ is a positive constant.

Received by the editors September 6, 1977.

AMS (MOS) subject classifications (1970). Primary 35J60, 35R05, 73E99.

Key words and phrases. Variational inequality, elastic set, plastic set, free boundary, reentrant corner, ridge.

${ }^{1}$ This work is partially supported by National Science Foundation Grants 7406375 A01 and MC575-21416 A01. 
It is well known that this problem has a unique solution $u$ [2], [9], [19]; furthermore, $u \in W^{2, p}(\tilde{Q})$ for any $p<\infty$, where $\tilde{Q}$ is any closed subset of $Q$ which does not contain vertices $V_{i}$. Further, it was proved in [7] that $u \in W^{2, \infty}\left(Q_{*}\right)$ for any compact subdomain $Q^{*}$ of $Q$.

Set

$$
d(x)=\text { distance from } x \text { to } \partial Q, \quad x=\left(x_{1}, x_{2}\right) \in \bar{Q} .
$$

Then [1], [19] the variational inequality (1.2) (with $K$ given by (1.1)) is equivalent to the same variational inequality with a different convex set $K$, namely,

$$
K=\left\{v \in H_{0}^{1}(Q), v \leqslant d\right\}
$$

The variational inequality (1.2), (1.3) (called the obstacle problem) is a more standard one; however the obstacle $d$ is not continuously differentiable, but only Lipschitz continuous. Thus we have here a situation where the solution is smoother than the obstacle.

The problem (1.1), (1.2) is called the elastic-plastic torsion problem. It arises when a long elastic bar with cross section $Q$ is twisted by an angle proportional to $\mu$. The set

$$
E=\{x \in \bar{Q} ;|\nabla u(x)|<1\}
$$

is the set of points where the cross section still remains elastic, and the set

$$
P=\{x \in \bar{Q} ;|\nabla u(x)|=1\}
$$

is the set of points where the material has become plastic due to the torsion. We refer to $E$ as the elastic set and to $P$ as the plastic set.

The part of the boundary of $E$ which is contained in $Q$ is called the free boundary; it will be denoted by $\Gamma$.

The derivation of the variational inequality from the physical problem can be found, for instance, in [11]. In a series of papers [13] [19] Ting has studied the variational inequality, with special attention to the case when $Q$ is a rectangle or a regular polygon. He also studied the set where $d$ is not smooth; this set is always elastic. The purpose of this paper is to study more thoroughly the free boundary.

In $\$ 2$ we define the concept of a ridge $R$ of a domain as the set of points where $d$ is not in $C^{1,1}$. We then characterize $R$ geometrically.

In $\S 3$ we prove that $R$ is contained in $E$, i.e., the ridge is elastic.

In $\S 4$ we study the case where $\mu \rightarrow \infty$ and prove that $E$ is contained in a neighborhood of $R$ of order of magnitude $1 / \mu$.

A vertex $V_{i}$ is called a reentrant corner if the opening of $Q$ at $V_{i}$ is larger than $\pi$. In $\$ 5$ we study the free boundary near a reentrant corner. We show, in fact, that a certain angular region with vertex $V_{i}$ is always plastic. 
In $\$ 6$ we show that any nonreentrant corner is contained in a $\bar{Q}$-neighborhood which is elastic.

In $\$ 7$ we study the free boundary near the boundary $\partial Q$, away from the vertices. We show that if $\sigma$ is a line segment or a circular arc contained in $\partial Q$ then the free boundary can intersect $\sigma$ in only a finite number of points.

In $\$ 8$ we consider domains which have an axis of symmetry. It is shown, under some restrictions, that $\Gamma$ consists of monotone arcs.

In $\S 9$ we illustrate the applicability of the results of $\S \S 2-8$ by studying in detail the free boundary for some special domains $Q$ : a rectangle, a regular polygon, a cross, and a cracked disc.

In $\$ 10$ we study the convexity of the free boundary in the case of a rectangle.

The rest of the paper is concerned with the elastic-plastic torsion problem in case $Q$ is multiply connected. Thus we suppose that $Q$ has "holes" $Q_{1}, \ldots, Q_{n}$. Let $Q^{*}=Q \cup\left(\cup_{i=1}^{n} \bar{Q}_{i}\right)$, i.e., $Q^{*}$ is a simply connected domain whose boundary is the exterior boundary $\partial_{e} Q$ of $Q$. Let

$$
K=\left\{v \in H_{0}^{1}\left(Q^{*}\right) ;|\nabla v| \leqslant 1, v=C_{i} \text { in } Q_{i}, 1 \leqslant i \leqslant n\right\}
$$

where $C_{i}$ are nonegative constants which vary with $v$. We replace (1.2) by the variational inequality

$$
\iint_{Q^{*}} \nabla u \cdot \nabla(v-u) \geqslant \mu \iint_{Q^{*}}(v-u) \quad \text { for every } v \in K ; u \in K,
$$

where $K$ is given by (1.7). Denote the (unique) solution by $u$, and set

$$
\begin{aligned}
C_{i}^{*} & =\left.u\right|_{Q_{i}} \\
\phi & =\max _{1<i<n}\left\{C_{i}^{*}-d_{i}\right\}, \\
\Phi & =\min \left\{\min _{1<i<n}\left\{C_{i}^{*}+d_{i}\right\}, d_{e}\right\}
\end{aligned}
$$

where $d_{i}(x)=$ distance from $x$ to $Q_{i}, d_{e}(x)=$ distance from $x$ to $\partial_{e} Q$. Let

$$
K=\left\{v \in H_{0}^{1}\left(Q^{*}\right), \phi \leqslant v \leqslant \Phi\right\} .
$$

Then [1], [19] $u$ is the unique solution of (1.7) with $K$ defined by (1.10).

In $\$ 11$ we generalize the results of $\$ \S 2-8$ to multiply connected domains. For simplicity we consider only the case $n=1$. In $\$ 12$ we prove that the set $\{u=\phi, u<\Phi\} \cap Q$ is generally nonempty. This answers a question raised by Lanchon [11].

2. The ridge of a domain. Roughly speaking the ridge of the domain $Q$ is the set of points $x^{0}$ in $Q$ such that the distance function $d(x)$ from $x$ to $\partial Q$ is not smooth enough in any neighborhood of $x^{0}$. Various definitions of a ridge were given by Ting [14], [19]. The motivation for these definitions is to be able to assert that the ridge lies in the elastic set of the domain. Therefore one 
would like to give as weak a definition as possible. Because of the recent regularity results of Caffarelli and Riviere [7] our definition will be weaker than the definitions of Ting.

Definition. The ridge $R$ of $Q$ is the set of all points $x^{0} \in Q$ such that $d(x)$ is not $C^{1,1}$ in any neighborhood of $x^{0}\left(C^{1,1}\right.$ means Lipschitz continuous first derivatives).

The purpose of this section is to geometrically characterize the set $R$.

We recall that throughout this paper (except for $\$ \S 11,12) Q$ is a simply connected bounded domain whose boundary consists of $C^{3}$ disjoint arcs $S_{1}, \ldots, S_{m}$ such that the terminal end-point $V_{i}$ of $S_{i}$ is the initial end-point of $S_{i+1}\left(1 \leqslant i \leqslant m, S_{m+1}=S_{1}\right)$. We denote by $\alpha_{i}$ the angle formed by $S_{i}$ and $S_{i+1}$ at $V_{i}$, which opens into $Q$. If $\alpha_{i} \geqslant \pi$ we call $V_{i}$ a reentrant corner, if $\alpha_{i}>\pi$ we call it a strict reentrant corner. If $\alpha_{i}<\pi$ then $V_{i}$ is called a nonreentrant corner.

LEMMA 2.1. If $d\left(x^{0}\right)=\left|x^{0}-y\right|=\left|x^{0}-z\right|$ where $y$ and $z$ are two different points on $\partial Q$, then $d(x)$ is not differentiable at $x^{0}$.

Proof. Along some interval (initiating at $x^{0}$ ) of the ray $\overrightarrow{x y^{\prime}}, d$ is linear with derivative -1 . The same is true for $\overrightarrow{x^{0} z}$. Thus

$$
\frac{\partial d}{\partial l_{1}}\left(x^{0}\right)=-1, \quad \frac{\partial d}{\partial l_{2}}\left(x^{0}\right)=-1
$$

where $l_{1}, l_{2}$ are the directions of $\overrightarrow{x y}, \overrightarrow{x^{0}} z$; these directions do not coincide. If $d(x)$ is differentiable at $x^{0}$ then $\left|\nabla d\left(x^{0}\right)\right|=1$ and therefore $l_{1}$ and $l_{2}$ must both coincide with the direction of $\nabla d\left(x^{0}\right)$, which is impossible.

Notation. $R_{0}$ is the set of all points $x^{0} \in Q$ such that $d\left(x^{0}\right)=\left|x^{0}-y\right|=$ $\left|x^{0}-z\right|$ for at least two distinct points $y, z$ on $\partial Q$.

LEMMA 2.2. Let $x^{0} \in Q \backslash R_{0}$ and denote by $y^{0}=y\left(x^{0}\right)$ the nearest point on $\partial Q$ to $x^{0}$. Denote by $z^{0}$ the center of the osculating circle at $y^{0}$. (We assume that $y^{0}$ is not a vertex.) Then $d$ is $C^{1,1}$ in a neighborhood of $x^{0}$ if and only if $x^{0} \neq z^{0}$.

Proof. Suppose first that $x^{0} \neq z^{0}$ and that $x^{0}$ lies on the ray $\overrightarrow{y^{0} z^{0}}$. Denote by $l$ the straight line containing $x^{0}, y^{0}$. Let $z_{-\varepsilon}, z_{\varepsilon}$ denote the points on $l$ such that

$$
\left|z_{-\varepsilon}-y^{0}\right|=\left|z^{0}-y^{0}\right|-\varepsilon \equiv r_{-\varepsilon}, \quad\left|z_{\varepsilon}-y^{0}\right|=\left|z^{0}-y^{0}\right|+\varepsilon=r_{\varepsilon},
$$

where $\varepsilon$ is positive and small, and let $r_{0}=\left|z^{0}-y^{0}\right|$. Denote by $C_{-\varepsilon}, C_{\varepsilon}, C_{0}$ the circles with centers $z_{-\varepsilon}, z_{\varepsilon}, z^{0}$ and radii $r_{-\varepsilon}, r_{\varepsilon}, r_{0}$ respectively, and denote by $d_{-\varepsilon}, d_{\varepsilon}, d_{0}$ the distance functions with respect to the domains $C_{-\varepsilon}, C_{\varepsilon}$ and $C_{0}$ respectively.

Notice now that, since $x^{0} \neq z^{0}, x^{0}$ must lie between $y^{0}$ and $z^{0}$ on $l$; 
otherwise there would be points on $\partial Q$, sufficiently close to $y^{0}$ whose distance to $x^{0}$ is smaller than $\left|x^{0}-y^{0}\right|$. Take a point $x_{*}$ between $x^{0}$ and $z_{-\varepsilon}$, and denote by $G_{\varepsilon}$ a sector with axis $\overrightarrow{x_{*} y^{0}}$ and small opening $\gamma_{\varepsilon}$; it contains a neighborhood $N_{\varepsilon}$ of $x^{0}$.

We can choose $\gamma_{e}$ so that

$$
d_{-\varepsilon} \leqslant d \leqslant d_{\varepsilon} \text { in } G_{\varepsilon} \cap Q .
$$

Notice that

$$
d_{-\varepsilon}=d=d_{\varepsilon} \quad \text { on } G_{\varepsilon} \cap Q \cap l .
$$

Choose orthogonal coordinates $(\xi, \eta)$ in $G_{\varepsilon}$ with the origin at $z_{\varepsilon}$. Then

$$
d_{\varepsilon}(\xi, \eta)=r_{\varepsilon}-\left(\xi^{2}+\eta^{2}\right)^{1 / 2} \text { in } G_{\varepsilon} \cap Q
$$

Therefore

$$
\begin{aligned}
\frac{\partial}{\partial \eta} d_{\varepsilon} & =-\frac{\eta}{\left(\xi^{2}+\eta^{2}\right)^{1 / 2}}, \\
\frac{\partial^{2}}{\partial \eta^{2}} d_{\varepsilon} & =-\frac{\xi^{2}}{\left(\xi^{2}+\eta^{2}\right)^{3 / 2}}
\end{aligned}
$$

It follows that

$$
\left|\frac{\partial^{2}}{\partial \eta^{2}} d_{\varepsilon}\right| \leqslant \frac{1}{\left(\xi^{2}+\eta^{2}\right)^{1 / 2}}=\frac{1}{r_{\varepsilon}-d_{\varepsilon}},
$$

or, in terms of $\kappa_{\varepsilon}=1 / r_{\varepsilon}=$ curvature of $C_{\varepsilon}$,

$$
\left|\frac{\partial^{2}}{\partial \eta^{2}} d_{\varepsilon}\right|<\frac{\kappa_{\varepsilon}}{1-\kappa_{\varepsilon} d_{\varepsilon}} .
$$

Taking, in particular, $\eta$ to be the direction normal to $l$, so that $\eta=0$ on $l$, we get from (2.4),

$$
\begin{aligned}
& \frac{\partial^{2}}{\partial \eta^{2}} d_{\varepsilon}=-\frac{1}{\xi}=-\frac{\kappa_{\varepsilon}}{1-\kappa_{\varepsilon} d_{\varepsilon}} \\
& \frac{\partial^{2}}{\partial \xi^{2}} d_{\varepsilon}=-\frac{\eta^{2}}{\left(\xi^{2}+\eta^{2}\right)^{3 / 2}}=0
\end{aligned}
$$

on $G_{\varepsilon} \cap Q \cap l$; hence

$$
\Delta d_{\varepsilon}=-\kappa_{\varepsilon} /\left(1-\kappa_{\varepsilon} d_{\varepsilon}\right) \text { on } G_{\varepsilon} \cap Q \cap l .
$$

Formulas similar to (2.3)-(2.6) hold for $d_{-\varepsilon}$.

Now, since

$$
\begin{aligned}
d_{\varepsilon}-d_{-\varepsilon} & >0 \quad \text { in } G_{\varepsilon} \cap Q, \\
& =0 \quad \text { on } G_{\varepsilon} \cap Q \cap l,
\end{aligned}
$$


we must have $\nabla\left(d_{\varepsilon}-d_{-\varepsilon}\right)=0$ on $G_{\varepsilon} \cap Q \cap l$. The second derivative of $d_{\varepsilon}-d_{-\varepsilon}$ in any direction $\eta$ can be computed by (2.4) and the corresponding formula for $d_{-\varepsilon}$. It follows that this second derivative is bounded by $O(\varepsilon)$. Since $\eta$ is an arbitrary direction, also the second mixed derivatives of $d_{\varepsilon}-d_{-\varepsilon}$ are bounded by $O(\varepsilon)$.

Since $d_{-\varepsilon} \leqslant d \leqslant d_{\varepsilon}$, we deduce from the above that $\nabla\left(d-d_{0}\right)=0$ on $G_{\varepsilon} \cap Q \cap l$, and that the limit of the second difference quotient $\Delta_{h}^{2}\left(d-d_{0}\right)$ on $G_{\varepsilon} \cap Q \cap l$ tends to zero as $h \rightarrow 0$. Notice that the derivatives of $d_{0}$ can be computed as in (2.3), (2.4) with $\varepsilon=0$.

The above analysis is valid for any $x$ in some neighborhood $W$ of $x^{0}$ (independent of $\varepsilon$ ) and, moreover, the limit of $\Delta_{h}^{2}\left(d-d_{0}\right)$ is uniform with respect to $x$ in $W$. Hence, by a standard theorem in calculus, $d \in C^{2}$ in $W$ and its second derivatives can be computed from (2.4) with $\varepsilon=0$; in particular,

$$
\Delta d=-k /(1-k d) \text { for any } x \in W,
$$

where $k$ is the curvature at the point $y=y(x)$ nearest to $x$ on $\partial Q$.

If $x^{0}$ does not lie on the ray $\overrightarrow{y^{0} z^{0}}$ then the above proof is valid with $d_{e}(\xi, \eta)$ given by $\left(\xi^{2}+\eta^{2}\right)^{1 / 2}-r_{e}$. In this case $d \in C^{2}$ in a neighborhood of $x^{0}$ and, in (2.7), $k$ is negative.

Suppose next that $z^{0}=x^{0}$. Let $x_{\varepsilon}$ be points on $l$ such that $\left|x_{\varepsilon}-y^{0}\right|=\mid x^{0}$ $-y^{0} \mid-\varepsilon$. For each $x_{\varepsilon}$ the above analysis is valid and, in particular, by (2.7),

$$
\Delta d\left(x_{\varepsilon}\right)=-k /\left(1-k d\left(x_{\varepsilon}\right)\right) \text {. }
$$

Since $d\left(x_{\varepsilon}\right) \rightarrow d\left(x^{0}\right)=1 / k$ as $\varepsilon \rightarrow 0$, we get

$$
\Delta d\left(x_{\varepsilon}\right) \rightarrow-\infty \quad \text { if } \varepsilon \rightarrow 0 .
$$

Hence $d$ cannot be in $C^{1,1}$ in any neighborhood of $x^{0}$.

We shall next extend Lemma 2.2 to the case where $y^{0}$ is a vertex.

LEMMA 2.3. Let $x^{0} \in Q \backslash R_{0}$ and denote by $y^{0}=y\left(x^{0}\right)$ the nearest point on $\partial Q$ to $x^{0}$. Suppose $y^{0}$ is a vertex (it is then, necessarily, a reentrant corner). Denote by $z^{1}, z^{2}$ the centers of the osculating circles corresponding to the two arcs of $\partial Q$ which meet at $y^{0}$. Then, $d$ is in $C^{1,1}$ in a neighborhood of $x^{0}$ if and only if $x^{0} \neq z^{1}$ and $x^{0} \neq z^{2}$.

Proof. Denote $l^{i}$ the straight line containing $y^{0}, z^{i}$ and denote by $\Sigma$ the open subset of $Q$ bounded by $l^{1} \cup l^{2}$. Then $x^{0}$ must belong to $\bar{\Sigma}$. If $x^{0} \notin l^{1} \cup l^{2}$ then it lies in $\Sigma$ and $d(x)=\left|x-y^{0}\right|$ for all $x$ in a neighborhood $W$ of $x^{0}$, so that $d$ is in $C^{2}(W)$.

If $x^{0} \in l^{1}$ and $x^{0} \neq z^{1}$ then the analysis of the previous proof is valid on one side of $l^{1}$, i.e. on the side $\Sigma_{0}$ which is disjoint to $\Sigma$. On the other hand $d(x)=\left|x-y^{0}\right|$ for $x \in \bar{\Sigma}, x$ near $x^{0}$, so that $d$ is in $C^{2}$ there. Thus it remains to show that the first derivatives of $d$ from both sides of $l^{1}$ coincide on $l^{1}$. 
This is of course true for the derivative of $d$ along $l^{1}$. Further, since this derivative is equal to 1 , in absolute value, and since $|\nabla d|=1$ on both sides of $l^{1}$, the normal derivatives of $d$ (from both sides of $l^{1}$ ) are equal to zero and thus coincide.

If $x^{0} \in l^{1}$ and $x^{0}=z^{1}$ then the last part of the proof of Lemma 2.2 can be carried out on one side of $l^{1}$, showing that $\Delta d\left(x_{e}\right) \rightarrow-\infty$ along an appropriate sequence of points $x_{e}, x_{e} \rightarrow x^{0}$. Thus $d$ is not in $C^{1,1}$ in any neighborhood of $x^{0}$.

Finally, the case where $x^{0} \in l^{2}$ can be handled in the same way as the case where $x^{0} \in l^{1}$.

Notation. A point $x^{0}$ in $Q$ is said to belong to the set $R_{1}$ if there is precisely one point $y^{0} \in \partial Q$ such that $d\left(x^{0}\right)=\left|x^{0}-y^{0}\right|$ and if $x^{0}$ is the center of the osculating circle at $y^{0}$ (or a center of one of the two osculating circles at $y^{0}$, if $y^{0}$ is a vertex).

Thus, Lemmas 2.2, 2.3 assert that if $x^{0} \in Q \backslash R_{0}$ then $d$ is in $C^{1,1}$ in some neighborhood of $x^{0}$ if and only if $x^{0} \notin R_{1}$.

From Lemmas 2.1-2.3 we obtain:

THEOREM 2.4. $R=R_{0} \cup R_{1}$.

REMARK. If there are no reentrant corners then $R_{1} \subset \bar{R}_{0}$, so that $R=\bar{R}_{0}$.

3. The ridge is elastic. Consider the variational inequality (1.1), (1.2). By [9], the solution is unique and it belongs to $W^{2, p}(\tilde{Q})$ for any $p<\infty$ and for any closed subdomain $\tilde{Q}$ (provided $\partial Q$ does not contain reentrant corners), and by [7], it belongs to $W^{2, \infty}\left(Q_{0}\right)$ (which is the same as $C^{1,1}\left(Q_{0}\right)$ ) for any closed subdomain of $Q_{0}$ of $Q$. By [1], the solution coincides with the solution of (1.2), (1.3) provided $\partial Q$ is sufficiently smooth; the same is true for our present domain $Q$, as follows by approximating it by a decreasing sequence of domains $Q_{j}$ with smooth boundary (cf. [7]). The following estimate was proved in [7]:

$$
\left|u_{x_{i} x_{j}}(x)\right| \leqslant C(\mu+1 / d(x)) \quad(x \in Q)
$$

where $C$ is a constant independent of $Q$ and $\mu$.

Recall that the sets $E$ and $P$ defined in (1.4), (1.5) are called the elastic and plastic sets, respectively, and the boundary of $E$ intersected with $Q$ is called the free boundary; it will be denoted by $\Gamma$.

It is easy to verify that

$$
\begin{aligned}
& E \cap Q=\{x \in Q ; u(x)<d(x)\}, \\
& P \cap Q=\{x \in Q ; u(x)=d(x)\} .
\end{aligned}
$$

Indeed, if $u\left(x^{0}\right)=d\left(x^{0}\right), x^{0} \in Q$, then since $u(x)-d(x) \leqslant 0$ in a neighborhood of $x^{0}$, we must have $\nabla(u-d)=0$ at $x^{0}$, so that $x^{0} \in P$. To complete the proof, we suppose that $u(y)<d(y)$ for some $y \in Q$, and show 
that $|\nabla u(y)|<1$. If this is not true, then consider the component $M$ of the set $\{u<d\}$ containing $y$. Let $l$ be the direction of $\nabla u$ at $y$, i.e., $|\nabla u(y)|$ $=\partial u(y) / \partial l$. Then $\partial u(y) / \partial l=1$. On the boundary of $M|\nabla u / \partial l|<1$ and therefore, by the strong maximum principle applied to $\partial u / \partial l, \partial u(x) / \partial l<1$ at $x=y$, a contradiction.

From (3.2), (3.3) it follows that $\Gamma$ is the part of the boundary of the set $\{u<d\}$ which lies in $Q$.

LEMMA 3.1. Let $x^{0} \in P, d\left(x^{0}\right)=\left|x^{0}-y^{0}\right|$ for some $y^{0} \in \partial Q$. Then $u(x)=$ $d(x)$ for all $x$ on the line segment l connecting $x^{0}$ to $y^{0}$.

Indeed, since $\partial d / \partial l=1, \partial u / \partial l \leqslant 1$ and $u=d$ at both end-points of $l$, we must have $\partial u / \partial l=1$ on $l$, that is, $u=d$ on $l$.

Lemma 3.1 is due to Ting [18].

THEOREM 3.2. $R \subset E$, that is, the ridge is elastic.

Proof. Let $x^{0} \in R$. We have to show that $x^{0} \in E$, that is, $u\left(x^{0}\right)<d\left(x^{0}\right)$. We suppose $u\left(x^{0}\right)=d\left(x^{0}\right)$ and derive a contradiction.

If $x^{0} \in R_{0}$ then

$$
d\left(x^{0}\right)=\left|x^{0}-y^{0}\right|=\left|x^{0}-y^{1}\right| \quad \text { where } y^{0} \in \partial Q, y^{1} \in \partial Q, y^{0} \neq y^{1} .
$$

By Lemma 3.1 it follows that

$$
u=d \text { on the line segments } l^{i} \text { connecting } x^{0} \text { to } y^{i} \text {. }
$$

The argument given in the proof of Lemma 2.1 now shows that $u$ is not differentiable at $x^{0}$, which is absurd.

If $x^{0} \in R_{1}$ then denote by $y^{0}$ the nearest point to $x^{0}$ on $\partial Q$, i.e., $\left|x^{0}-y^{0}\right|$ $=d\left(x^{0}\right)$, and let $l$ denote the line segment connecting $x^{0}$ to $y^{0}$. Suppose first that $y^{0}$ is not a vertex. We have

$$
\begin{aligned}
u=d \quad \text { on } l \quad(\text { by Lemma 3.1), } \\
\nabla(u-d)=0 \quad \text { on } l \quad(\text { since } u-d \leqslant 0 \text { in } Q), \\
\frac{\partial^{2} d(x)}{\partial \eta^{2}} \rightarrow-\infty \quad \text { if } x=\left(\xi^{0}, \eta\right) \rightarrow x^{0}=\left(\xi^{0}, 0\right)
\end{aligned}
$$

where $(\xi, \eta)$ is a coordinate system with $\eta$ normal to $l$ and $\xi=0$ along $l$. Since $u \leqslant d$, we must then also have

$$
\partial^{2} u(x) / \partial \eta^{2} \rightarrow-\infty
$$

where $\partial^{2} u / \partial \eta^{2}$ is taken in the sense of the limit of the difference quotient of the first derivative $\partial u / \partial \eta$. Thus $\partial u / \partial \eta$ is not Lipschitz continuous at $x^{0}$, which is impossible.

If $y^{0}$ is a vertex then it is necessarily a reentrant corner and the above analysis is valid from one side of $l$. 
4. $\mu \rightarrow \infty$. The set $E$ decreases monotonically with $\mu$. We shall estimate its size as $\mu \rightarrow \infty$.

Notation. $\delta(x)=$ distance from $x$ to the ridge; $x \in Q$.

THEOREM 4.1. There exists a positive constant $C$ independent of $\mu$ such that

$$
E \cap Q \subset\{x \in Q ; \delta(x)<C / \mu\} \text {. }
$$

Proof. Denote by $B_{h}(x)$ the disc with center $x$ and radius $h$. We claim:

$$
\text { if } B_{\rho}(\bar{x}) \subset E \text { then } \rho<\min \left\{2(d(\bar{x}) / \mu)^{1 / 2}, 4 / \mu\right\} \text {. }
$$

Indeed, applying the maximum principle to the harmonic function

$$
w=u+\mu\left(|x-\bar{x}|^{2}-\rho^{2}\right) / 4 \text { in } B_{\rho}(\bar{x}),
$$

it follows that

$$
w(\bar{x}) \geqslant\left.\inf w\right|_{\partial B_{\rho}(\bar{x})}>0,
$$

so that

$$
\mu \rho^{2} / 4<u(\bar{x})<d(\bar{x})
$$

Next, let

$$
\lambda=\inf _{\partial B_{\rho}(\bar{x})} u .
$$

Since $|\nabla u| \leqslant 1, u-\lambda \leqslant \rho$ in $B_{\rho}(\bar{x})$. Applying the maximum principle to

$$
\tilde{w}=(u-\lambda)+\mu\left(|x-\bar{x}|^{2}-\rho^{2}\right) / 4 \text { in } B_{\rho}(\bar{x})
$$

we get $\tilde{w}(\bar{x})>0$, which gives, since $u(\bar{x})-\lambda \leqslant \rho$,

$$
\rho>\mu \rho^{2} / 4 \text {. }
$$

The assertion (4.2) now follows from (4.3), (4.4).

Let $x^{0}$ be any point in $Q \backslash R$. Then there exists a unique point $y^{0} \in \partial Q$ such that $\left|x^{0}-y^{0}\right|=d\left(x^{0}\right)$. Suppose $y^{0}$ is not a vertex and denote by $z^{0}$ the center of the osculating circle at $y^{0}$. Then $x^{0}$ lies in the interior of the line segment $l$ connecting $y^{0}$ to $z^{0}$. By (2.7) and $\left|y^{0}-z^{0}\right|=1 / k$,

$$
\Delta d\left(x^{0}\right)=-1 /\left|x^{0}-z^{0}\right| \text {. }
$$

Now, if $d\left(z^{0}\right)=\left|z^{0}-y^{0}\right|$ then $z^{0} \in R$. If $d\left(z^{0}\right)<\left|z^{0}-y^{0}\right|$ then, by continuity, there exists a point $x_{*}$ in the interval $\overline{x^{0} z^{0}}$ for which $d\left(x_{*}\right)$ is attained at both $y^{0}$ and at another point on $\partial Q$; thus $x_{*} \in R$. In both cases we conclude that

$$
\delta\left(x^{0}\right) \leqslant\left|x^{0}-z^{0}\right|
$$

and together with (4.5),

$$
\Delta d\left(x^{0}\right) \geqslant-1 / \delta\left(x^{0}\right) \text { if } x^{0} \in Q \backslash R,
$$


provided $y^{0}$ is not a vertex. If $y^{0}$ is a vertex (and then, necessarily, a reentrant corner) then $d(x)=\left|x-y^{0}\right|$ from one side of $l$, so $\Delta d=1 / d \geqslant 0$ from that side. We thus conclude that

$$
\Delta d(x) \geqslant-1 / \delta(x) \text { a.e. in } Q \backslash R,
$$

where $\Delta d$ is taken in the sense of weak derivatives, say.

For any positive constant $C$ we denote by $Q_{C}$ the subset of $Q$ consisting of all points $x$ such that $\delta(x)>C / \mu$. By (4.7),

$$
\Delta d \geqslant-\mu / C \text { in } Q_{C} .
$$

Let $A$ be a sufficiently large positive constant, to be determined later on. Suppose there is a point $x^{0}$ in $Q_{A} \cap E$. Let

$$
h=\frac{1}{2} \min \left(\delta_{0}, d_{0}\right) \quad \text { where } \delta_{0}=\delta\left(x^{0}\right), d_{0}=d\left(x^{0}\right) .
$$

The function

$$
w=d-u-\left(1-\frac{2}{A}\right) \mu \frac{\left|x-x^{0}\right|^{2}}{4} \quad \text { in } B_{h}\left(x^{0}\right)
$$

satisfies $\Delta w \geqslant 0$ (by (4.8)) and $w\left(x^{9}\right)>0$. By the maximum principle we get

$$
\sup _{\partial B_{h}\left(x^{0}\right)} w>0 \text {. }
$$

Hence, if $A>4$, there exists a point $x^{1} \in \partial B_{h}\left(x^{9}\right)$ such that

$$
d\left(x^{1}\right)-u\left(x^{1}\right)>h^{2} \mu / 8 .
$$

It then follows, by [5], that

$$
B_{r}\left(x^{1}\right) \subset E
$$

provided

$$
r^{2}|u|_{C^{1,1}\left(B_{h}\left(x^{0}\right)\right)}=h^{2} \mu / C
$$

where $C$ is a constant independent of $\mu, h, A$. Using (3.1) we can take $r$ to be given by

$$
r^{2}=C h^{2} /\left(1+1 /\left(\mu d_{0}\right)\right)
$$

with another constant $C$.

Since, by (4.2), we must have $r<4 / \mu$, we get, after using (4.9),

$$
\left[\min \left(\delta_{0}, d_{0}\right)\right]^{2} \leqslant C\left(1 / \mu^{2}+1 / \mu^{3} d_{0}\right)
$$

where $C$ is independent of $\mu, h_{0}, A$; we take $C>1$.

Let $K=d_{0} \mu$. If $\min \left(\delta_{0}, d_{0}\right)=d_{0}$ and $K>1$ then it follows from (4.10) that

$$
\frac{K^{2}}{\mu^{2}} \leqslant \frac{C}{\mu^{2}}\left(\frac{1}{K}+1\right), \text { i.e., } K \leqslant \sqrt{2 C}
$$


Therefore

$$
\begin{aligned}
& \text { if } d\left(x^{0}\right)>\sqrt{2 C} / \mu \text { then } \delta\left(x^{0}\right)<d\left(x^{0}\right) \\
& \text { and } \delta\left(x^{0}\right)<C_{0} / \mu \text {, where } C_{0} \text { is independent of } A, \mu \text {. }
\end{aligned}
$$

We claim that the inequality

$$
d\left(x^{0}\right) \leqslant \sqrt{2 C} / \mu
$$

cannot occur if $A$ is sufficiently large. Indeed, suppose (4.12) holds and let $y^{0}$ be the nearest point to $x^{0}$ on $\partial Q$. There are points $\bar{x}$ on the ray $\overrightarrow{y^{0} x^{0}}$ so that $x^{0}$ lies between $y^{0}$ and $\bar{x}$ and

$$
\frac{\sqrt{2 C}}{\mu}<d(\bar{x})<\frac{\sqrt{2 C}+1}{\mu}, \quad d(\bar{x})=\left|\bar{x}-y^{0}\right| .
$$

(We use here the fact that $\delta\left(x^{0}\right)>A / \mu$ and $A$ is sufficiently large.) Hence we can apply (4.11) to such a point $\bar{x}$ and conclude that

$$
\delta(\bar{x})<C_{0} / \mu
$$

which is impossible since

$$
\delta(\bar{x}) \geqslant \delta\left(x^{0}\right)-\left|\bar{x}-x^{0}\right| \geqslant \frac{A}{\mu}-\frac{\sqrt{2 C}+1}{\mu}>\frac{C_{0}}{\mu}
$$

if $A$ is sufficiently large.

Since (4.11) holds and (4.12) does not hold, we deduce that if $x^{0} \in Q_{A} \cap E$ then $\delta\left(x^{0}\right) \leqslant C_{0} / \mu$. But this is again impossible if $A>C_{0}$. Thus the set $Q_{A} \cap E$ is empty if $A$ is sufficiently large, and (4.1) follows.

REMARK. The estimate (4.1) is sharp. Consider, for example, a rectangle $Q$ (which is not a square) with sides parallel to the axes. With a suitable choice of the origin, the interval $\gamma=\{(x, 0) ; a<x<b\}$ is a part of the horizontal ridge and $d(x, y)=c-|y|, c>0$. Suppose $\left(x_{0}, h\right) \in P, a<x_{0}<b, h>0$. On one hand we have

$$
I \equiv-h^{-2}\left[u\left(x_{0}, 3 h\right)+u\left(x_{0},-h\right)-2 u\left(x_{0}, h\right)\right] \leqslant C_{0}|u|_{1,1} \leqslant C \mu
$$

where $C_{0}, C$ are constants independent of $\mu$. On the other hand,

$$
\begin{gathered}
u\left(x_{0}, 3 h\right) \leqslant c-3 h, \\
u\left(x_{0},-h\right) \leqslant c-h, \\
u\left(x_{0}, h\right)=c-h,
\end{gathered}
$$

so that

$$
I \geqslant 2 h / h^{2}=2 / h \text {. }
$$

It follows that $h>2 /(C \mu)$. Thus $E$ must contain any $(A / \mu)$-neighborhood of any closed subinterval $\gamma_{0}$ of $\gamma$, where $A$ is a constant depending only on $\gamma_{0}$. 
5. The free boundary near a reentrant corner. Suppose $V_{i}$ is a strict reentrant corner. Thus the arcs $S_{i}, S_{i+1}$ of $\partial Q$ which meet at $V_{i}$ form an angle $\alpha_{i}$ (in $Q$ ) such that $\alpha_{i}>\pi$. Denote by $\gamma_{1}, \gamma_{2}$ the tangent lines to $S_{i}, S_{i+1}$, respectively, at $V_{i}$. We choose the coordinate system $(x, y)$ so that $V_{i}$ is the origin and $\gamma_{1}, \gamma_{2}$ form angles $-\alpha, \alpha$ with the positive $x$-axis. Thus $\alpha_{i}=2 \alpha, \alpha>\pi / 2$. Set $\beta=\alpha-\pi / 2$.

In the sequel we shall also use polar coordinates $(r, \theta)$.

THEOREM 5.1. There exists a continuous curve $r=\rho(\theta)$ defined for $-\beta<\theta$ $<\beta$ such that $\rho(\theta)>0$ and such that, for all $(r, \theta)$ with $-\beta<\theta<\beta$,

$$
(r, \theta) \in P \quad \text { if } r \leqslant \rho(\theta) .
$$

We shall need the following lemma.

LEMMA 5.2. Let $D=\left\{(r, \theta) ; 0<r<r_{0},|\theta|<\gamma\right\}, \gamma>\pi / 2$ and denote by $E, P$ the elastic and plastic sets of $D$. Then there is a segment $\sigma=\{(r, \theta)$; $\left.0<r<r_{1}, \theta=0\right\}$ which is contained in $P$.

Proof. Consider the function

$$
u_{\theta}=x u_{y}-y u_{x}
$$

in the region $E^{+}=E \cap\{\theta>0\}$. This function is harmonic (since $\Delta u_{\theta}=$ $\left.(\Delta u)_{\theta}=(-\mu)_{\theta}=0\right)$ and vanishes on the part of $\partial E^{+}$where $r=r_{0}$ (since $u=0$ if $r=r_{0}$ ) and on $\theta=0$ (by symmetry). On the segment $\theta=\gamma, u_{\theta} \leqslant 0$ (since $u=0$ on $\theta=\gamma, u \geqslant 0$ elsewhere). Finally, on the free boundary part of $\partial E^{+}, u_{\theta}=d_{\theta} \leqslant 0$ since $d$ is decreasing in $\theta$ along any circular arc in $E^{+}$. By the maximum principle we then conclude that $u_{\theta} \leqslant 0$ in $E^{+}$.

In the smaller set

$$
E_{0}^{+}=E \cap\left\{0<\theta<\gamma-\pi / 2, r<r_{0} / 2\right\}
$$

$P$ is a subgraph $r \leqslant f(\theta)$ (by Lemma 3.1), but $f(\theta)$ may a priori be equal to zero for some values of $\theta$. Since $u_{\theta} \leqslant 0$ in $E^{+}$and since $d(r, \theta)=r$ in $E_{0}^{+}$, we have

$$
(u-d)_{\theta} \leqslant 0 \text { in } E_{0}^{+} .
$$

Thus $f(\theta)$ is monotone decreasing in $\theta, 0<\theta<\gamma-\pi / 2$.

Now, if the assertion of the lemma is not true then $f(0)=0$ and, from the monotonicity of $f$ it follows that $f(\theta)=0$ if $0 \leqslant \theta \leqslant \gamma-\pi / 2$. By symmetry we also have $f(\theta)=0$ if $-\gamma+(\pi / 2) \leqslant \theta \leqslant 0$. Let

$$
\begin{aligned}
& G=\left\{(r, \theta) ;|\theta|<\gamma-\pi / 2,0<r<r_{0} / 2\right\}, \\
& B=D \cap\left\{0<r<r_{0} / 2\right\} .
\end{aligned}
$$

Then $G$ is contained in $E$ and therefore $\Delta u=-\mu$ in $G$. On the other hand, if $x \in B \backslash G$ then either $x \in E$, in which case $\Delta u=-\mu<0$, or $x \in P$ in which case a.e. $\Delta u \leqslant \Delta d=0$ (since $d$ is linear in $B \backslash G)$. Thus 


$$
\Delta u \leqslant 0 \text { a.e. in } B \text {. }
$$

Consider the harmonic function

$$
v=c r^{\pi / 2 \gamma} \cos (\pi \theta / 2 \gamma) \quad(c>0)
$$

in $B$. Suppose

$$
\partial u / \partial v \neq 0 \text { at }\left(r_{0} / 2, \pm \gamma\right)
$$

where $\nu$ is the normal. Then, if $c$ is sufficiently small, $v<u$ on $\partial B$. Since also, by (5.2), $\Delta v \geqslant \Delta u$ a.e. in $B$, the maximum principle gives $u \geqslant v$ in $B$. In particular,

$$
u(r, 0) \geqslant v(r, 0)=c r^{\pi / 2 \gamma} \text {. }
$$

Since however $u(r, 0) \leqslant d(r, 0)=r$ and $(\pi / 2 \gamma)<1$, we'get a contradiction if $r \rightarrow 0$. This completes the proof of the lemma in case (5.3) is satisfied.

The assertion (5.3) at $\left(r_{0} / 2, \gamma\right)$ is true if there exists a $D$-neighborhood of $\left(r_{0} / 2, \gamma\right)$ which is elastic. Otherwise, there is a plastic segment initiating at a point $\left(r_{0}^{*} / 2, \gamma\right)$ and orthogonal to $\theta=\gamma$, where $r_{0}^{*}$ is arbitrarily close to $r_{0}$. We then have

$$
\frac{\partial u}{\partial \nu}\left(\frac{r_{0}^{*}}{2}, \gamma\right)=1
$$

A similar situation holds with respect to the point $\left(r_{0} / 2,-\gamma\right)$. Thus by replacing, if necessary, $B$ by a domain

$$
B^{*}=\left\{0<r<r_{0}^{*} / 2,0<\theta<\gamma\right\} \cup\left\{0<r<r_{0}^{* *} / 2,-\gamma<\theta<0\right\},
$$

we can continue as before to deduce that $u \geqslant v$ in $B^{*}$ and, consequently, derive a contradiction.

Proof of Theorem 5.1. Take any direction $\theta=\theta_{0}$ in $(-\beta, \beta)$ and construct a domain $D$ as in Lemma 5.2, so that it is contained in $Q$, its axis of symmetry is $\theta=\theta_{0}$, and

$$
\gamma=\pi / 2+\left(\beta-\left|\theta_{0}\right|\right) / 2
$$

Denote by $u_{D}$ the solution $u$ corresponding to the domain $D$. Along the ray $\theta_{0}$

$$
u_{D} \leqslant u \quad \text { (by the monotonicity of the obstacles), }
$$

and also $u \leqslant d=r$ if $r$ is sufficiently small. By Lemma 5.2,

$$
u_{D}\left(r, \theta_{0}\right)=r \text { if } r \text { is sufficiently small, }
$$

say $r<\bar{r}$. It follows that $u\left(r, \theta_{0}\right)=r$ if $r<\bar{r}$, i.e., $\left(r, \theta_{0}\right) \in P$. The positive number $\bar{r}$ can be taken independently of $\theta_{0}$, provided $\theta_{0}$ is restricted to a closed subinterval of $(-\beta, \beta)$, and this completes the proof.

COROLlaRY 5.3. The estimate (3.1) is sharp near $\partial Q$ in the sense that

$$
d(x) \Delta u(x)=1
$$

if $x$ approaches a strict reentrant corner along the bisector. 
Indeed, by Theorem 5.1 we have, at such points

$$
\Delta u=\Delta d=1 / d .
$$

6. The free boundary near a nonreentrant corner. Let $V_{i}$ be a vertex of $Q$ which is not a reentrant corner. Thus, the arcs $S_{i}, S_{i+1}$ form an angle at $V_{i}$ (in $Q)$ of size $\alpha_{i}<\pi$.

THEOREM 6.1. There exists a neighborhood $N$ of $V_{i}$ such that $\bar{Q} \cap N$ is contained in $E$.

Proof. Consider first the case where $S_{i}, S_{i+1}$ are line segments. Introduce polar coordinates with center at $V_{i}$ so that, for some neighborhood $W$ of $V_{i}, W \cap Q$ is the sector

$$
G=\left\{(r, \theta) ; 0<r<r_{0},-\beta<\theta<\beta\right\}, \quad \alpha_{i}=2 \beta .
$$

Then, in $G$,

$$
\Delta u(x)= \begin{cases}-\mu & \text { if } x \in E \\ \Delta d & \text { if } x \in P \text { (a.e.) }\end{cases}
$$

we use here the fact that since $u$ and $d$ are in $C^{1,1}$ in $Q \backslash R$, they are twice differentiable a.e. in $Q \backslash R$, and hence a.e. in $G$ (if $r_{0}$ is sufficiently small).

Since $Q \cap R \subset E$ and $\Delta d$ is smooth with bounded second derivatives in $G \backslash R$,

$$
\Delta u \text { is bounded in } G \text {. }
$$

Suppose now that

$$
\beta>\pi / 4 \text {. }
$$

Consider the function

$$
v=r^{\pi / 2 \beta} \cos \frac{\pi \theta}{2 \beta}+\left(y^{2}-x^{2} \tan ^{2} \beta\right)
$$

in $G$. It satisfies

$$
\Delta v=1-\tan ^{2} \beta<0 \text { in } G .
$$

Also $v=0$ on $\theta= \pm \beta$ and $v>0$ if $r=r_{0},|\theta|<\beta$ provided $r_{0}$ is sufficiently small, and the normal derivative of $v$ is different from zero at $\left(r_{0}, \pm \beta\right)$.

Recalling (6.1) we conclude, by the maximum principle, that for some positive and sufficiently large $C$,

$$
u \leqslant C v \text { in } G .
$$

Hence

$$
u(r, \theta)<C d r^{\nu}<\frac{1}{2} d \quad(\nu=\pi / 2 \beta-1>0)
$$

if $r$ is sufficiently small, say $r<\delta$. Thus $\bar{G} \cap\{r<\delta\} \subset E$. 
Consider next the case that $\beta \leqslant \pi / 4$. We shall prove that

$$
\{(r, \theta) \in \bar{G},-\beta \leqslant \theta \leqslant 0, r<\delta\} \subset E
$$

if $\delta$ is sufficiently small; the proof for $0 \leqslant \theta \leqslant \beta$ is similar.

Let $Q_{1}$ be a domain containing $Q$, such that $Q_{1}$ has a vertex $V_{i}$ and $Q_{1} \cap\left\{r<r_{0}\right\}$ is a sector of opening $\gamma>\pi / 2$, with $\gamma<\pi$, and such that the two arcs of $\partial Q_{1}$ that meet at $V_{i}$ are line segments and one of them lies on $\theta=-\beta$. By comparison, the solution $\boldsymbol{w}$ of the variational inequality corresponding to $Q_{1}$ satisfies $w \geqslant u$ in $Q$.

By the proof given above

$$
w \leqslant \frac{1}{2} d_{Q_{1}} \text { in some } Q_{1} \text {-neighborhood of } V_{i},
$$

where $d_{Q_{1}}$ is the distance function $d$ for $Q_{1}$. Since $d_{Q_{1}}=d$ if $-\beta \leqslant \theta<0, r$ small enough, we conclude that $u<d$ in this set, and (6.4) follows.

We have thus completed the proof of the theorem in case $S_{i}, S_{i+1}$ are line segments. To prove the theorem for general arcs $S_{i}, S_{i+1}$, we transform $Q \cap W\left(W\right.$ a neighborhood of $\left.V_{i}\right)$ into a sector $G$ as above by a conformal mapping $f$, where the opening $2 \beta$ of $G$ is the same as the opening $\alpha_{i}$ of $Q$ at $V_{i}$.

Denote by $z=g(\tilde{z})$ the inverse of $\tilde{z}=f(z)$. Then

$$
g(\tilde{z})=\left(h\left(\tilde{z}^{\alpha}\right)\right)^{1 / \alpha}, \quad \alpha=\pi / 2 \beta>1,
$$

where $\eta=h(\zeta)$ maps a domain bounded by two smooth curves $S_{1}^{1}, S_{2}^{1}$ and forming an angle $\pi$ at their common end-point into $\operatorname{Im} \eta>0$, with $S_{1}^{1}, S_{2}^{1}$ mapped into $\eta>0, \eta<0$. The inverse function of $h$, say $k$, is in $C^{1+\varepsilon}, \varepsilon>0$, because, by [3, Appendix], its real part is in $C^{1+\varepsilon}$. Thus, $h$ is also in $C^{1+\varepsilon}$ if we can show that the gradient of $\operatorname{Re} k$ at the origin is $\neq 0$.

Let

$$
v=\rho \sin \theta-\rho^{1+\delta} \cos (1+\delta) \theta .
$$

Then $v<0$ along $S_{1}^{1} \cup S_{2}^{1}$ if $\delta<1 / \alpha$, and $|\nabla v|=1$ at the origin. By comparison, $\operatorname{Re} k \geqslant c v$ for some $c>0$. Hence $\nabla(\operatorname{Re} k) \neq 0$ at the origin.

Having proved that $h$ is $C^{1+\varepsilon}$ for some $\varepsilon>0$, it now follows that $g^{\prime}(\tilde{z})$ is a bounded function.

Setting $\tilde{u}(\tilde{z})=u(z)$ where $z=g(\tilde{z})$, we have

$$
\Delta \tilde{u}=\left|g^{\prime}\right|^{2} \Delta u \quad \text { is bounded. }
$$

Hence we can apply to $\tilde{u}$ the preceding special case and conclude that

$$
\tilde{u} \leqslant C \tilde{d} \tilde{r}^{\nu} \text { near the origin, }
$$

where $\nu>0, \tilde{d}$ is the distance function for the transformed region, and $\tilde{r}=|\tilde{z}|$. Since $\tilde{d}<C d, \tilde{r}<C r$, we get $u<\frac{1}{2} d$ in some $Q$-neighborhood of $V_{i}$, and the proof is complete. 
COROLLARY 6.2. Let $W$ be any neighborhood of the set of reentrant corners. If $\mu$ is sufficiently small then $P \subset W$.

Proof. Let $w$ be the solution of

$$
\Delta w=-\mu \text { in } Q, \quad w=0 \text { on } \partial Q .
$$

By comparison, $u \leqslant \mu w$ in $Q$. Now, the exterior ball property holds at all the points of $\partial Q$ other than the reentrant corners; hence barriers exist. Therefore, for any subdomain $Q_{0}$ of $Q$ such that $\bar{Q}_{0}$ does not contain reentrant corners of $Q$,

$$
w \leqslant C d ; \quad C \text { constant depending on } Q_{0} \text {. }
$$

It follows that

$$
u \leqslant \mu C d \leqslant \frac{1}{2} d \text { in } Q_{0},
$$

provided $2 \mu C<1$, so that $\bar{Q}_{0}$ is contained in $E$; the proof of the corollary is complete.

Suppose $V_{i}$ is a strict reentrant corner and $S_{i}, S_{i+1}$ are line segments. Let $\beta=\alpha_{i} / 2$. If $\tan ^{2} \beta<1$ then we can still employ the comparison function $v$ as in (6.3). More precisely, since $\Delta d=1 / d$ or 0 near $V_{i}$, we have $\Delta u>-\mu$ in $G$ (instead of (6.1)). Therefore we actually have $u<C \mu v$ where $C$ is independent of $\mu\left(0<\mu<1\right.$, say). It follows that $u<d$ as long as $2 \mu C r^{\pi / 2 \beta}<r$, i.e., as long as

$$
r>c \mu^{(2 \beta-\pi) / 2 \beta} \quad(c>0) .
$$

Thus the plastic set in a neighborhood of $V_{i}$ is contained in a $c \mu^{\left(\alpha_{i}-\pi\right) / \alpha_{i}}$ neighborhood of $V_{i}$.

The same assertion is true for general arcs $S_{i}, S_{i+1}$, by using the conformal mapping $f$ as in the proof of Theorem 6.1.

If $\tan ^{2} \beta>1$ then we can repeat the argument with the function

$$
v=r^{\pi / 2 \beta} \cos \frac{\pi \theta}{2 \beta}-\left(y^{2}-x^{2} \tan ^{2} \beta\right) .
$$

Finally, if $\tan ^{2} \beta=1$, we replace $-\left(y^{2}-x^{2} \tan ^{2} \beta\right)$ in (6.5) by the solution $\zeta$ of

$$
\begin{aligned}
\Delta \zeta & =-1 \quad \text { in } G, \\
\zeta & =0 \text { on } \partial G .
\end{aligned}
$$

This solution is positive and bounded above by $\mathrm{Cr}^{4 / 3}$ (as seen by considering $\zeta-x^{2} / 2$ and using the conformal mapping $z \rightarrow z^{2 / 3}$ ). Hence $v \leqslant C r^{2 / 3}$ (with another constant $C$ ) and we can proceed as before.

We have thus proved: 
COROLlaRY 6.3. Let $N$ be a small neighborhood of a strict reentrant corner $V_{i}$. Then the plastic set $P \cap N$ is contained in a

$$
c \mu^{\left(\alpha_{i}-\pi\right) / \alpha_{i}} \text { neighborhood of } V_{i} \quad(c>0)
$$

for all $\mu$ sufficiently small.

7. The behavior of $\Gamma$ near $\partial Q$. In this section we study the behavior of $\Gamma$ near arcs of $\partial Q$ which are either linear or circular.

THEOREM 7.1. Suppose $\sigma_{0}$ is an open line segment contained in $\partial Q$. Then $\Gamma$ intersects any closed subarc $\sigma$ of $\sigma_{0}$ in, at most, a finite number of points, say $q_{1}, \ldots, q_{k}$, and the tangents to $\Gamma$ and $\sigma$ at each point $q_{i}$ coincide.

Recall [6] that $\Gamma$ is analytic.

Proof. We introduce a coordinate system such that

$$
\sigma_{1}=\left\{(x, 0): a_{1}<x<a_{2}\right\}, \quad \text { where } \sigma \subset \sigma_{1} \subset \bar{\sigma}_{1} \subset \sigma_{0},
$$

and the rectangle

$$
N=\left\{(x, y) ; a_{1} \leqslant x \leqslant a_{2}, 0 \leqslant y \leqslant b\right\}
$$

is contained in $Q$.

If there exists a $\bar{Q}$-neighborhood of $\left(a_{1}, 0\right)$ contained in $E$, we take $\bar{a}_{1}=a_{1}$. Otherwise, there is a plastic interval $x=\bar{a}_{1}, 0<y<h$ for $\bar{a}_{1}$ arbitrarily close to $a_{1}$. Therefore, either there is a point $\left(\bar{a}_{1}, \bar{b}_{1}\right)$ on $\Gamma$ with $\bar{b}_{1}<b$ or the line segment $x=\bar{a}_{1}, 0 \leqslant y \leqslant b_{1}$ is plastic. Similarly we define $\bar{a}_{2}$ and $\bar{b}_{2}$. Set

$$
\begin{aligned}
& \tilde{\boldsymbol{\sigma}}=\left\{(x, 0) ; \bar{a}_{1}<x<\bar{a}_{2}\right\}, \\
& \tilde{N}=\left\{(x, y) ; \bar{a}_{1} \leqslant x \leqslant \bar{a}_{2}, 0 \leqslant y \leqslant b\right\} .
\end{aligned}
$$

We choose $b$ so small that $d(x, y)=y$ if $(x, y) \in \tilde{N}$. It follows (by Lemma 3.1) that the free boundary in $\tilde{N}$ is given by $y=\phi(x), 0<\phi(x) \leqslant b$ for $x$ in a subset $\Sigma$ of $\left(\bar{a}_{1}, \bar{a}_{2}\right)$. What we have to show is that $\Sigma$ consists of a finite number of intervals; the analyticity of $\Gamma$ (and moreover of $\phi[6]$ ) implies that $\Sigma$ consists of at most a countable number of intervals.

Every interval $J_{i}$ of $\Sigma$ gives rise to a "loop" $\Gamma_{i}$ of the free boundary $\Gamma$. Suppose the number of $J_{i}$ is infinite. The height of $\Gamma_{i}$, as $i \rightarrow \infty$, must go to zero. For, as $i \rightarrow \infty$, meas $\cup{ }_{k=i}^{\infty} J_{k} \rightarrow 0$ and, by applying the maximum principle to $\tilde{u}-\mu(y-\varepsilon)^{2} / 2(\tilde{u}=y-u, \varepsilon>0)$ in a subset of $E$ bounded by arcs of $\Gamma_{i}, \Gamma_{j}, y=0$ and a horizontal line segment $l$ connecting the arcs in $\tilde{N} \cap\{y>0\}$, we find (after letting $\varepsilon \rightarrow 0$ ) that $\max _{l} \tilde{u}>\mu h^{2} / 2$ where $h$ is the $y$-coordinate on $l$. Since $u \in C^{1,1}$ in the interior, $\max _{l} \tilde{u}<c|l|^{2} \rightarrow 0$ if $i, j \rightarrow \infty(|l|=$ length of $l)$; it follows that $h \rightarrow 0$. Thus, the height of $\Gamma_{i}$ tends to zero as $i \rightarrow \infty$.

It follows that there is only a finite number of loops which intersect $y=b$. 
Set $z=(u-d)_{x}$. Since $\Gamma$ intersects $\tilde{\sigma}$ at an infinite number of points, $z(x, \varepsilon)$, for $\bar{a}_{1}<x<\bar{a}_{2}$, changes sign $\lambda(\varepsilon)$ times, where $\lambda(\varepsilon) \rightarrow \infty$ if $\varepsilon \rightarrow 0$. Since $z$ is harmonic in $\tilde{N} \backslash P$, by using level curves $z=$ const. starting on $y=\varepsilon$, we find that $z$ must also change sign an infinite number of times on $\partial \tilde{N} \cap E ;$ since $z=0$ on $\tilde{\sigma}$, these changes of sign occur on the set $M=(\partial \tilde{N} \backslash$ б) $\cap \bar{E}$.

Now, from the analyticity of $\Gamma$ it follows that $z$ is analytic in $E$ up to the boundary $\Gamma$. Since, as proved above, there is only a finite number of intersection points of $\Gamma$ with $\partial \tilde{N} \backslash \tilde{\sigma}$ we find (using also the construction of $\bar{a}_{1}, \bar{a}_{2}$ above) that $z$ is analytic in $\tilde{N} \backslash P$ up to $M$; therefore $z$ cannot change sign on $M$ an infinite number of times; a contradiction.

In order to complete the proof of the theorem, it remains to show that if $y=\phi(x), \alpha<x<\beta$, is a loop of $\Gamma$ with end-points in $\tilde{\sigma}$ then $\phi^{\prime}(\alpha)=\phi^{\prime}(\beta)$ $=0$ [and a similar result in case only one end-point lies in $\tilde{\sigma}$ ]. The proof is similar to the proof of a corresponding result for the dam problem [4].

Indeed, by analyticity, $z_{x x}$ and its tangential derivative along $M$ change sign at most a finite number of times. This fact can be used to show, by the arguments of [4], that only a finite number of maximum domains and inflection domains can alternate on $\tilde{\sigma}$ and, similarly, only a finite number of inflection domains and maximum inflection domains can alternate on $\tilde{\sigma}$.

With this in mind, the proof that $\phi^{\prime}(\alpha), \phi^{\prime}(\beta)$ exist and are equal to zero is the same as for Lemma 8.2 of [4].

THEOREM 7.2. Suppose $\sigma_{0}$ is an open circular arc contained in $\partial Q$. Then $\Gamma$ intersects any closed subarc $\sigma$ of $\sigma_{0}$ in at most a finite number of points.

The proof is similar to the proof of Theorem 7.1, if we work with the function

$$
z=\partial(u-d) / \partial \theta
$$

which is harmonic in an $E$-neighborhood of the circular arc.

REMARK. For a general analytic arc $\sigma$ lying in $\partial Q$, one is tempted to repeat the above proof with

$$
z=e^{\lambda}\left(u_{x} d_{y}-u_{y} d_{x}\right)
$$

However in order to derive for $z$ an elliptic equation (for a suitably chosen function $\lambda$ ) we are led to a system of equations whose solution exists if and only if the arc is either linear or circular.

8. Domains with an axis of symmetry. We consider domains

$$
Q=\{(x, y) ;-g(y)<x<g(y), a<y<b\}
$$

where $g(y)$ is not necessarily a continuous function.

Let $Q^{+}=Q \cap\{x>0\}$. 
LEMMA 8.1. $u_{x} \leqslant 0$ in $Q^{+}$.

Proof. Let $(x, y) \in Q^{+}$and denote by $\left(x_{1}, y_{1}\right)$ a point on $\partial Q$ nearest to $(x, y)$. By symmetry, $x_{1} \geqslant 0$. Denote by $\vec{l}$ the vector initiating at $(x, y)$ and terminating at $\left(x_{1}, y_{1}\right)$, and by $\vec{l}_{h}(h>0)$ the vector which is equal to $\vec{l}$ but initiates at $(x+h, y)$. Since $\left(x_{1}, y_{1}\right) \in \partial Q, x_{1} \geqslant 0$, the end-point $\left(x_{1}+h, y_{1}\right)$ of $\vec{l}_{h}$ does not belong to $Q$. It follows that

$$
d(x+h, y) \leqslant\left\|\vec{l}_{h}\right\|=\|\vec{l}\|=d(x, y) .
$$

Thus,

$$
d_{x} \leqslant 0 \text { in } Q^{+} \text {. }
$$

Let $E^{+}=E \cap Q^{+}$. By symmetry, $u_{x}=0$ on $x=0$. On the part of $\partial E^{+}$ which belongs to the boundary of $Q, u_{x} \leqslant 0$ (because $u=0$ on $\partial Q, u \geqslant 0$ elsewhere). On the part of $\partial E^{+}$which belongs to the free boundary,

$$
u_{x}=d_{x} \leqslant 0 \text {, by (8.2). }
$$

It follows, by the maximum principle, that $u_{x} \leqslant 0$ in $E^{+}$.

THEOREM 8.2. Let $Q$ be given by (8.1) and let

$$
g\left(y_{0}-0\right)<g\left(y_{0}+0\right) \text { for some } a \leqslant y_{0}<b .
$$

Set $J=\left\{\left(x, y_{0}\right) ; g\left(y_{0}-0\right)<x<g\left(y_{0}+0\right)\right\}$ and let $Q_{0}$ be a subdomain of $Q$ such that $\partial Q_{0} \cap \partial Q \subset J$ and $d(x, y)=y-y_{0}$ if $(x, y) \in Q_{0}$. Then $\Gamma \cap Q_{0}$, if nonempty, is given by

$$
y=h(x)
$$

where $h(x)$ is strictly monotone decreasing and analytic.

Proof. In $Q_{0}$ we have $(d-u)_{x}=-u_{x} \geqslant 0$, by Lemma 8.1. It follows that $E \cap Q_{0}$ is a $y$-subgraph. By Lemma 3.1 this set is also an $x$-subgraph. Therefore, $\Gamma \cap Q_{0}$ is given by (8.3) where $h(x)$ is monotone decreasing. The continuity and strict monotonicity of $h$ follow by a standard argument of unique continuation, and the analyticity of $h$ was already established, in general, for the free boundary, by Caffarelli and Riviere [6].

Let $V_{i}$ be a strict reentrant corner and choose the coordinate axes so that the tangent ray to $S_{i}$ (i.e., the limit of rays from $V_{i}$ to $(x, y) \in S_{i}$, as $\left.(x, y) \rightarrow V_{i}\right)$ is the negative real axis, and the ray perpendicular to $S_{i}$ at $V_{i}$ and going in $Q$ is the positive $y$-axis.

If $s$ is the length parameter along $S_{i}$ (with $s=0$ for $V_{i}$ ), then the free boundary in a $Q$-neighborhood of $S_{i}$ which lies in $\{x<0\}$ and is given, whenever existing, by

$$
x=f(s), y=g(s) \quad((f(s), g(s)) \in Q)
$$

(as follows by Lemma 3.1). 
Set

$$
G=\left\{(r, \theta) ; 3 \pi / 2-\alpha_{i}<\theta<\pi / 2,0<r<r_{0}\right\} .
$$

By Theorem 5.1, there is a $G$-neighborhood of $V_{i}$ which is plastic. Therefore, the free boundary in $G$ is given by

$$
r=\rho(\theta), \quad \rho(\theta)>0
$$

for $\theta$ in a subset of $\left(3 \pi / 2-\alpha_{i}, \pi / 2\right)$.

We shall now assume that the functions in (8.4), (8.5) in fact exist near $s=0$ and $\theta=\pi / 2$ respectively, that is,

$\Gamma$ is given by (8.4) for $0<s<s_{0}$ and by (8.5)

$$
\text { for } \theta_{0}<\theta<\pi / 2 \text {, for some } s_{0}>0, \theta_{0}<\pi / 2 \text {. }
$$

From [6] it follows that $f(s), g(s)$ are analytic for $0<s<s_{0}$ and $\rho(s)$ is analytic for $\theta_{0}<\theta<\pi / 2$.

THEOREM 8.3. Let (8.6) hold; then

$$
\begin{aligned}
& (f(s), g(s)) \text { is continuous at } s=0, \\
& \rho(\theta) \text { is continuous at } \theta=\pi / 2, \\
& (f(0), g(0)) \text { coincides with }(\rho(\pi / 2), \pi / 2) .
\end{aligned}
$$

Proof. If $(f(s), g(s))$ has two points of accumulation, we can obtain a contradiction by the argument used in the proof of Theorem 7.1 to deduce that the heights $\Gamma_{i} \rightarrow 0$ as $i \rightarrow \infty$. Thus (8.7) is valid. The proof of (8.8) is similar. To prove (8.9), suppose the assertion is false. Let $J$ be the line segment on the $y$-axis representing the jump from $(f(0), g(0))$ to $(\rho(\pi / 2), \pi / 2)$. Then the function $u$ satisfies

$$
\begin{aligned}
u=y & \text { along } J \text { (by Lemma 3.1), } \\
u_{x}=0 & \text { along } J
\end{aligned}
$$

since $u_{x}=d_{x}=0$ on $J$. Also $\Delta u=-\mu$ on a one-sided neighborhood of $J$. By uniqueness for the Cauchy problem with data on $J$ we conclude that

$$
u-y+\mu x^{2} / 2 \equiv 0 \text { in this neighborhood, }
$$

and therefore also in $E$, which is impossible.

9. Examples. We shall apply the results of $\S \S 2-8$ to special domains $Q$.

9.1. $Q$ is a rectangle. Let

$$
Q=\{(x, y) ;-a<x<a,-b<y<b\}, \quad b<a .
$$

Then the ridge consists of the segment

$$
l_{0}=\{(x, 0) ;|x|<a-b\}
$$

and the four segments $l_{i}$ joining end-points of $l_{0}$ with the nearest vertices; $l_{i}$ is the segment lying in the $i$ th quadrant. 
By Theorem 3.2, the free boundary $\Gamma=\Gamma_{\mu}$ stays away from the ridge, and, by Theorem 6.1, it stays away from the vertices. Theorem 8.2 implies that the free boundary in the region bounded by $l_{1}, l_{2}, l_{0}$ and $y=b$ is given by

$$
y=f_{\mu}(x) \text { for }-a_{\mu}<x<a_{\mu}, 0 \leqslant a_{\mu}<a,
$$

and $f_{\mu}(x)=f_{\mu}(-x), f_{\mu}(x)$ is strictly monotone increasing for $0 \leqslant x<a_{\mu}$. This function is also analytic in $x,-a_{\mu}<x<a_{\mu}$. Notice that $a_{\mu}=0$ means that the free boundary does not exist. By Corollary 6.2 , this is indeed the case if $\mu$ is sufficiently small. In fact, $a_{\mu}$ is monotone increasing in $\mu$, and the smallest $\mu_{0}$ such that $a_{\mu}>0$ if $\mu>\mu_{0}$ is given by

$$
\mu_{0} \partial u_{0}(0, b) / \partial y=-1
$$

where $u_{0}$ is the solution of

$$
\Delta u_{0}=-1 \text { in } Q, \quad u_{0}=0 \text { on } \partial Q .
$$

By Theorem 4.1, $\Gamma_{\mu}$ exists if $\mu$ is sufficiently large. We shall now make more precise the estimate on $E$ given in Theorem 4.1.

Take any point $\left(x^{0}, f_{\mu}\left(x^{0}\right)\right), a-b<x^{0}<a_{\mu}$, and denote by $\delta$ the distance from this point to the ridge. The triangle $\Delta$ whose sides lie on the lines $x=x^{0}, y=f_{\mu}\left(x^{0}\right), l_{1}$ is contained in $E$. We can put a disc $S$ of radius $\delta / 2 \sqrt{2}$ inside $\Delta$ and, by applying (4.2), we conclude that $\delta<8 \sqrt{2} / \mu$. The same estimate can be obtained in case $0<x^{0}<a-b$. Thus

$$
E \subset 8 \sqrt{2}-\mu \text {-neighborhood of the ridge. }
$$

By Theorem 7.1,

$$
f_{\mu}^{\prime}\left( \pm a_{\mu}\right)=0 .
$$

The behavior of the free boundary in the other quadrants is similar.

We finally note that $u_{x x} \leqslant 0, u_{y y} \leqslant 0$ in $E$, as seen by applying the maximum principle to $u_{x x}, u_{y y}$ in $E$.

The monotonic behavior of $f_{\mu}$ was proved by Ting [13], [15] using Steiner symmetrization.

9.2. $Q$ is a regular polygon. Let $Q$ be a regular polygon with $n$ vertices. The ridge consists of $n$ line segments $l_{i}$ connecting the vertices $V_{i}$ to the center 0 . It is sufficient to analyze the free boundary $\Gamma$ in the triangle $\Delta_{1}$ formed by $l_{1}, l_{2}$. We take the coordinate axes so that $0=(0,0)$ and

$$
\begin{aligned}
& l_{1}: y=-\beta x, \quad 0<x<a, \\
& l_{2}: y=\beta x, \quad-a<x<0 .
\end{aligned}
$$

As in the case of the rectangle one can show that $\Gamma \cap \Delta_{1}$ is given by

$$
y=f_{\mu}(x), \quad-\beta a<f(x)<0 \text { for }-a_{\mu}<x<a_{\mu}
$$

and $f_{\mu}(-x)=f_{\mu}(x), f_{\mu}(x)$ is strictly increasing and analytic for $-a<x<0$, and $f_{\mu}^{\prime}\left( \pm a_{\mu}\right)=0$. Further, (9.4) is valid. 
Introduce oblique derivatives $(\xi, \eta)$ by

$$
\xi=y+\beta x, \quad \eta=y-\beta x .
$$

Notice that $l_{1}$ lies on the axis $\xi=0$ and $l_{2}$ lies on the axis $\eta=0$. We shall prove:

$$
\begin{aligned}
& \Gamma \cap \Delta_{1} \text { can be represented in the forms } \\
& \xi=\phi(\eta) \quad\left(\eta \in J_{1}\right) \text { and } \eta=\psi(\xi) \quad\left(\xi \in J_{2}\right)
\end{aligned}
$$

where $J_{1}, J_{2}$ are intervals and $\phi, \psi$ are analytic in the interior of $J_{1}, J_{2}$, respectively, and continuous at the end-points.

Denote by $l_{12}$ the line segment joining $(0,0)$ to $(0,-\beta a)$. Let $\Delta_{1}^{+}$be the triangle two of whose sides are $l_{12}, l_{2}$. Next let $\Delta_{2}^{-}$be the triangle two of whose sides are $l_{2}$ and $l_{23}$, the bisector of $l_{2}, l_{3}$ (from $(0,0)$ to $\partial Q$ ). Denote by $\Delta$ the interior of the closure of $\Delta_{1}^{+} \cup \Delta_{2}^{-}$.

The function $d$ is linear in $\Delta_{1}^{+} \cup \Delta_{2}^{-}$and $d_{\eta}$ is continuous across $l_{2}$. Hence the function $z=(u-d)_{\eta}$ is harmonic in $\Delta \cap E$. On the part of $\partial(\Delta \cap E)$ which belongs to $\Gamma, z=0$. On the part of $\partial(\Delta \cap E)$ which belongs to $\partial Q$ we clearly have $z \leqslant 0$ (since $z=0$ on $\partial Q, z \leqslant 0$ in $Q$ ). On $\partial(\Delta \cap E) \cap l_{12}$ we have

$$
\begin{aligned}
& (u-d)_{x}=u_{x}-d_{x}=0-0=0, \\
& (u-d)_{y}=u_{y}-d_{y}=u_{y}-1 \leqslant 0,
\end{aligned}
$$

so that $z_{\eta} \leqslant 0$. Similarly $z_{\eta} \leqslant 0$ on $\partial(\Delta \cap E) \cap l_{23}$.

Applying the maximum principle to $z$ in $\Delta \cap E$, we deduce that $z<0$ in $\Delta$. Thus, in particular,

$$
(u-d)_{\eta}<0 \text { in } \Delta_{1}^{+}=\Delta_{1} \cap\{x>0\} .
$$

It follows that every line $\eta=c$ intersects $\Gamma \cap \Delta_{1}^{+}$in at most one point. Using the fact that $\Gamma \cap \Delta_{1} \cap\{x \leqslant 0\}$ is represented in the form $y=f_{\mu}(x), f_{\mu}$ increasing, it is clear that any line $\eta=c$ can intersect $\Gamma \cap \Delta_{1} \cap\{x<0\}$ in at most one point. Consequently $\Gamma \cap \Delta_{1}$ can be represented in the form $\xi=$ $\phi(\eta), \eta \in J_{1}$ where $J_{1}$ is an interval. The analyticity of $\phi$ follows from [6], and the continuity of $\phi$ at the end-points is proved in the same way as (8.7).

The results proved above have also been proved by Ting [15] using Steiner and circular symmetrizations.

9.3. $Q$ is a cross. We take $Q$ to have the shape of a cross with sides parallel to the $x$-axis and $y$-axis and with axes of symmetry coinciding with the $x$-axis and $y$-axis. Denote by $Q_{k}$ the intersection of $Q$ with the $k$ th quadrant. The ridge of $Q$ is designated by bold lines in the following figure: 


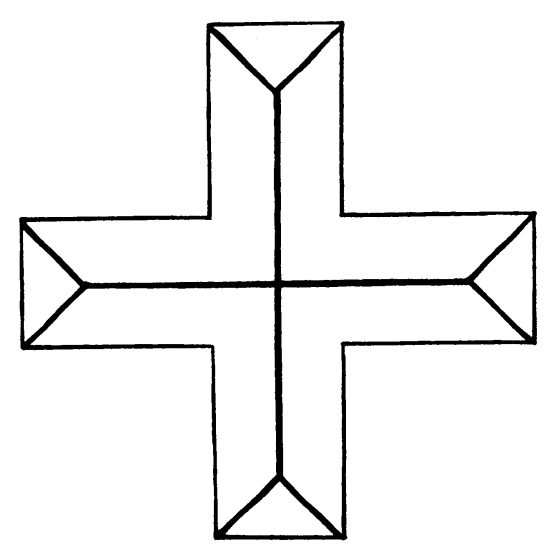

$Q_{4}$ is the union $\cup_{i=1}^{5} S_{i}$ where, for some $0<a<b$,

$S_{1}=\{-b<x<-b+a, x+b-a<y<0\}$ (triangle),

$S_{2}=\{-b<x<-a,-a<y<0\} \backslash S_{1}$ (trapeze),

$S_{3}=\{-a<x<0,-a<y<0\}$ (square),

$S_{4}$ and $S_{5}$ are the reflections with respect to $y=x$ of $S_{2}$ and $S_{1}$, respectively.

The free boundary $\Gamma$ in $S_{1}$ (if existing) is given by

$$
x=g_{1}(y), \quad g_{1} \text { monotone increasing; }
$$

this is proved as in the case of a rectangle, using Theorem 8.2.

The free boundary in $S_{2}$ (if existing) is given by

$$
y=g_{2}(x), \quad g_{2}(x) \text { monotone increasing }
$$

(by Theorem 8.2). The shape of $\Gamma$ in $S_{4}$ and $S_{5}$ is the same as in $S_{2}$ and $S_{1}$, respectively.

In order to study $\Gamma$ in the square $S_{2}$, consider the triangle $T$ bounded by the line $x=-a$ and the rays $y= \pm x$. Introduce polar coordinates $(r, \theta)$ with respect to $(-a,-a)$ as center, so that $\theta=0$ corresponds to the ray on $y=-a$ along which $x$ increases.

LEMMA 9.1. $u_{\theta} \leqslant 0$ in $T \cap E$.

Proof. Consider the harmonic function

$$
u_{\theta}=(x+a) u_{y}-(y+a) u_{x}
$$

in $T \cap E$. On the side $x=-a, u_{x} \geqslant 0$ by Lemma 8.1, so that $u_{\theta} \leqslant 0$. On the line $y=x, u_{\theta}=0$ by symmetry. On the line $y=-x, u_{x} \geqslant 0$ and $u_{y} \leqslant 0$ (by Lemma 8.1) so that $u_{\theta} \leqslant 0$. Finally, on the free boundary, $u_{\theta}=d_{\theta}$ and

$$
d_{\theta}=0 \text { in } T \cap\{y<0\}, \quad d_{\theta}<0 \text { in } T \cap\{y>0\}
$$

so that $u_{\theta} \leqslant 0$. Applying the maximum principle, the assertion of the lemma follows. 
From Lemma 9.1 it follows that the free boundary in $S_{3} \cap\{y>x\}$, if existing, is given by

$$
r=\rho(\theta), \quad \rho(\theta) \text { monotone decreasing. }
$$

By Theorem 5.1 and Theorem 3.2 it follows that, for $\pi / 4<\theta_{0}<\pi / 2$, $\Gamma \cap S_{3} \cap\{y>x\}$ is, in fact, intersected by the ray $\theta=\theta_{0}$ and $\rho\left(\theta_{0}\right)>0$. By Theorem 8.2, if $\rho(\pi / 2)>0$ then, and only then, the free boundary $y=g_{2}(x)$ (in (9.7)) does exist and $\left(-a, g_{2}(-a)\right)$ coincides with $(\pi / 2, \rho(\pi / 2))$. The shape of the free boundary in $S_{3} \cap\{y<x\}$ is symmetric (with respect to $y=x$ ) to that of $(9.8)$.

9.4. $Q$ is a cracked disc. Let $Q$ be a disc of radius $R$ with a segment along a radius removed. More specifically, let

$$
Q=\left\{(x, y) ; x^{2}+y^{2}<R^{2}\right\} \backslash \gamma, \quad \gamma=\{(0, y) ;-R \leqslant y \leqslant-\alpha\}
$$

where $\alpha \geqslant 0$. Set

$$
0=(0,0), \quad 0^{\prime}=(0,-\alpha) .
$$

Even though the two arcs of $\partial Q$ which meet at $\sigma^{\prime}$ coincide (except for orientation), the existence and regularity results of $\$ 1$ remain valid for the present domain $Q$.

We shall work with polar coordinates $(r, \theta)$ centered at 0 , and with polar coordinates $\left(r^{\prime}, \theta^{\prime}\right)$ centered at $0^{\prime}$.

The ridge of $Q$ is given by the bold-faced curves in the following figure:

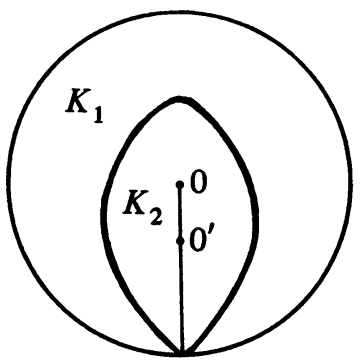

It divides $Q$ into two simply connected components $K_{1}$ and $K_{2}$, with $\partial K_{1}$ containing the circular part of $\partial Q$. Let $K_{21}=K_{2} \cap\{y>-\alpha\}, K_{22}=K_{2} \cap$ $\{y<-\alpha\}$. If $(x, y) \in K_{21}$ then the nearest point on $\partial Q$ to $(x, y)$ is 0 . If $(x, y) \in K_{22}$ then the nearest point on $\partial Q$ to $(x, y)$ lies on $\gamma$.

Set

$$
S_{1}=Q \cap\{x<0\}, \quad S_{2}=S_{1} \cap\{-R<y<-\alpha\} .
$$

Since $d=R-\left(x^{2}+y^{2}\right)^{1 / 2}$ and $d_{\theta^{\prime}}=x d_{y}-(y+\alpha) d_{x}$, we find that $d_{\theta^{\prime}}=$ $\alpha x /\left(x^{2}+y^{2}\right)^{1 / 2}$, so that

$$
d_{\theta^{\prime}} \leqslant 0 \text { in } S_{1}
$$


LEMMA 9.2. The following inequalities hold:

$$
\begin{array}{ll}
u_{\theta} \leqslant 0 & \text { in } S_{1} \cap E, \\
u_{\theta^{\prime}} \leqslant 0 & \text { in } S_{1} \cap E, \\
u_{y} \geqslant 0 & \text { in } S_{2} \cap E .
\end{array}
$$

PROOF. The proof of (9.11) follows by applying the maximum principle to $u_{\theta}$, noting that $u_{\theta}=d_{\theta} \leqslant 0$ on $\partial\left(S_{1} \cap E\right) \cap \Gamma, u_{\theta} \leqslant 0$ on $\partial S_{1} \cap \partial Q$, and $u_{\theta}=0$ on $\partial S_{1} \cap\{x=0\}$. The proof of (9.12) is the same, if we make use of (9.10). From (9.12) it follows that $u_{y} \geqslant 0$ on $\partial S_{2} \cap\{y=-\alpha\}$. Since $u_{y} \geqslant 0$ on the remaining part of $\partial\left(S_{2} \cap E\right)$, the assertion (9.13) follows by applying the maximum principle to $u_{y}$.

THEOREM 9.3. The free boundary in $S_{1}$ consists of three curves:

$\Lambda_{1}: r=\rho_{1}(\theta), \rho_{1}(\theta)$ monotone increasing in $\theta, \pi / 2 \leqslant \theta<\theta_{1}$ for some $\theta_{1}<3 \pi / 2$,

$\Lambda_{2}: r^{\prime}=\rho_{2}\left(\theta^{\prime}\right), \rho_{2}\left(\theta^{\prime}\right)$ monotone decreasing in $\theta^{\prime}, \pi / 2<\theta^{\prime} \leqslant \pi$,

$\Lambda_{3}: x=f(y), f(y)$ monotone decreasing in $y,-\alpha_{1}<y<-\alpha$ for some $\alpha_{1}<R$.

$\Lambda_{1}$ lies in $K_{1}$ and may be empty; $\Lambda_{2}$ lies in $K_{21}$ and is always nonempty, and $\Lambda_{3}$ lies in $K_{22}$ and may be empty. Further, $\Lambda_{3}$ is nonempty if and only if $\rho_{2}(\pi)>0$, and in that case $\Lambda_{2}$ and $\Lambda_{3}$ form a continuous curve.

The proof follows by using arguments as in the proof of Theorem 8.2, and also using Theorems 5.1 and $\$ 8.3$.

If $\alpha<0$ then in general one cannot expect the monotonic behavior of the free boundary as in Theorem 9.3, since the ridge is not monotone and, by Theorem 4.1, the free boundary "converges" to the ridge as $\mu \rightarrow \infty$.

10. Convexity properties of the free boundary for a rectangle. We consider again the case where $Q$ is the rectangle (9.1). Recall that the free boundary consists of at most four disjoint arcs $\sigma_{i}(1 \leqslant i \leqslant 4)$; $\sigma_{i}$ lies in both the $i$ th and $(i+1)$ th quadrants if $1 \leqslant i \leqslant 3$, and $\sigma_{4}$ lies in the fourth and first quadrants.

We shall assume for definiteness that all the four arcs are nonempty; otherwise the proofs given below have to be slightly changed.

We can represent $\sigma_{3}$ in the form

$$
y=f(x) \text { for }-\alpha<x<\alpha(0<\alpha<a)
$$

where $f(-x)=f(x) ; f(x)$ is strictly monotone increasing for $-\alpha<x<0$, it is analytic for $-\alpha<x<\alpha$, and $f^{\prime}( \pm \alpha)=0$.

THEOREM 10.1. The function $f(x)$, for $-\alpha<x<0$, has precisely one inflection point $-x_{0}$, that is,

$$
\begin{aligned}
& f^{\prime \prime}(x)<0 \text { if }-x_{0}<x<x_{0} \\
& f^{\prime \prime}(x)>0 \text { if }-\alpha<x<-x_{0} \text { or } x_{0}<x<\alpha .
\end{aligned}
$$


Proof. The proof uses some arguments developed in [4]. We recall that

$$
u_{y y}(x, f(x))=\frac{\mu}{1+\left(f^{\prime}(x)\right)^{2}}
$$

and $f^{\prime}(0)=0$. Hence

$$
u_{y y}(0, f(0))=u_{y y}(\alpha, f(\alpha))=\mu \quad(f(\alpha)=-b) .
$$

Suppose (10.1) is not true. Then there exist two points $0<x_{1}<x_{2}<\alpha$ such that $\left(f^{\prime}(x)\right)^{2}$ has a maximum at $x=x_{1}$ and at $x=x_{2}$. It follows that there is a point $\bar{x}, x_{1}<\bar{x}<x_{2}$, such that $\left(f^{\prime}(x)\right)^{2}$ has a minimum at $x=\bar{x}$.

Set

$$
\alpha_{i}=u_{y y}\left(x_{i}, f\left(x_{i}\right)\right), \quad \bar{\alpha}=u_{y y}(\bar{x}, f(\bar{x})) .
$$

Then

$$
\alpha_{i}<\bar{\alpha} \text {. }
$$

We now introduce the inflection domain $G_{i}$ with vertex $\left(x_{i}, f\left(x_{i}\right)\right)$, i.e., $G_{i}$ is the connected component in $E$ of the set $\left\{u_{y y}(x, y)<\alpha_{i}\right\}$ whose boundary contains $\left(x_{i}, f\left(x_{i}\right)\right)$. We also introduce the maximum inflection domain $N$ with vertex $(\bar{x}, f(\bar{x}))$, i.e., the connected component in $E$ of the set $\left\{u_{y y}(x, y)\right.$ $>\bar{\alpha}\}$.

By [8], [4], $u_{y y}$ cannot take local maximum or local minimum at any point of the free boundary which is contained in an arc of the form $y=\phi(x)$ (or $x=\psi(y)$ ) where $\phi($ or $\psi)$ is monotone. By the maximum principle, $u_{y y}$ must take its minimum in $\bar{G}_{i}$ on $\partial G_{i}$. Since $u_{y y}=\alpha_{i}$ on $\partial G_{i} \cap E$ and $u_{y y}=\mu$ on the horizontal part of the boundary of $Q$, we conclude that $\partial G_{i}$ must contain either a vertical part of $\partial Q\left(u_{y y}=0\right.$ at such points) or the points (one or two) where $y=0$ intersects $\sigma_{4}$ and $\sigma_{2}$ (at these points $u_{y y}=0$ ).

By symmetry with respect to both the $x$-axis and the $y$-axis it follows that $\partial G_{i}$ must contain

(i) either the lower vertical part $\lambda: x=a,-b<y<y_{0}$ of $\partial Q$ which lies in $E$,

(ii) or the point $p=\sigma_{4} \cap\{y=0\}$.

Since the maximum inflection domain $N$ lies between $G_{1}$ and $G_{2}$ and $f(x)$ is monotone for $x_{1}<x<x_{2}$, the maximum principle for $u_{y y}$ in $N$ implies that $\partial N$ must contain points which do not belong to either $E$ or the arc $\left\{(x, f(x)), x_{1}<x<x_{2}\right\}$; for otherwise $u_{y y}$ does not take its maximum in $\bar{N}$ on the boundary $\partial N$.

This last assertion together with (10.2) imply that $G_{1}$ and $G_{2}$ must be disjoint. Hence $\partial G_{1}$ contains the point $p$ and $\partial G_{2}$ contains the segment $\lambda$. But since $u_{y y}$ in $\bar{N}$ must take its maximum on $\partial N$, this maximum must be attained on the part of $\sigma_{4}$ which lies in the fourth quadrant. This is impossible since this part of $\sigma_{4}$ has the form $x=\psi(y)$ where $\psi$ is a monotone function. 
11. $Q$ is multiply connected. Let $Q$ be a multiply connected domain with "holes" $Q_{1}, \ldots, Q_{n}$, where each $\partial Q_{i}$ is piecewise $C^{3}$. Define $K$ by (1.6) where $Q^{*}=Q \cup\left(\cup_{i=1}^{n} \bar{Q}_{i}\right)$. Then the solution $u$ of the variational inequality (1.7) belongs to $W^{2, p}(\tilde{Q})$ for any $p<\infty$ and any closed subset $\tilde{Q}$ of $Q$ which does not include vertices of $\partial Q$ (provided $\partial Q$ satisfies the outside ball property) [2], [9], [19]. Further [2], [19], if $C_{i}^{*}=\left.u\right|_{Q_{i}}$ and if we define $\phi, \Phi$ by (1.8), (1.9), then $u$ is the unique solution of (1.7) where $K$ is defined by (1.10). Thus $u$ is the solution of a two-sided obstacle problem.

We recall [6] that $u \in W^{2, \infty}(\tilde{Q})$ for any compact subdomain $\tilde{Q}$ of $Q$; the estimate (3.1) is also valid.

From now on we shall assume that $n=1$, i.e., $Q$ has just one hole $Q_{1}$. However all the results extend to any finite number $n$ of holes.

Recall that $\partial_{e} Q$, the outer boundary of $Q$, consists of a finite number of curves $S_{1}, \ldots, S_{l}$. We denote by $V_{i}$ the common end-point of $S_{i}$ and $S_{i+1}$ (where $S_{l+1}=S_{1}$ ). Similarly, the boundary $\partial Q_{1}$ of $Q_{1}$ consists of a finite number of curves $T_{1}, \ldots, T_{l_{1}}$, such that the terminal point of $T_{i}$ and the initial point of $T_{i+1}$ coincide $\left(T_{l_{1}+1}=T_{1}\right)$; this common point is denoted by $W_{i}$.

Let $u$ be the solution of (1.6), (1.7) (with $m=1$ ) and define

$$
\begin{aligned}
C_{\mu} & =\left.u\right|_{Q_{1}} \\
C_{\infty} & =\operatorname{dist}\left(\partial Q_{1}, \partial_{e} Q\right), \\
d_{i}(x) & =\operatorname{dist}\left(x, Q_{1}\right) \quad\left(x \in \overline{Q^{*}}\right), \\
d_{e}(x) & =\operatorname{dist}\left(x, \partial_{e} Q\right) \quad\left(x \in \overline{Q^{*}}\right), \\
\phi(x) & =C_{\mu}-d_{i}(x), \\
\Phi(x) & =\min \left\{d_{e}(x), C_{\mu}+d_{i}(x)\right\} .
\end{aligned}
$$

Notice (since $|\nabla u| \leqslant 1$ ) that $C_{\mu} \leqslant C_{\infty}$, so that $\Phi \geqslant \phi$.

Definitions. The upper ridge $R^{+}$of $Q$ is the set of all points $x^{0} \in Q$ such that $\Phi(x)$ is not in $C^{1,1}$ in any neighborhood of $x^{0}$. The lower ridge $R^{-}$of $Q$ is the set of all points $x^{0} \in Q$ such that $\phi$ is not in $C^{1,1}$ in any neighborhood of $x^{0}$. The set $R=R^{+} \cup R^{-}$is called the ridge of $Q$.

Notice that these definitions depend on the value of the constant $C_{\mu}$. Denote by $R_{e}\left(R_{i}\right)$ the set of points $x^{0} \in Q$ such that $d_{e}\left(d_{i}\right)$ is not in $C^{1,1}$ in any neighborhood of $x^{0}$. Then we clearly have

$$
R^{+}=\left(R_{e} \cap\left\{d_{e}<C_{\mu}+d_{i}\right\}\right) \cup\left(R_{i} \cap\left\{d_{e}>C_{\mu}+d_{i}\right\}\right) \cup R_{0}^{+}
$$

where

$$
R_{0}^{+}=\left\{d_{e}=C_{\mu}+d_{i}\right\} \cap Q
$$


and

$$
R^{-}=R_{i}
$$

Definition. The upper plasticity $P^{+}$is the set of points in $Q$ where $u=\Phi$. The lower plasticity $P^{-}$is the set of points in $Q$ where $u=\phi$.

We claim (cf. (3.2), (3.3)) that

$$
P \cap Q \equiv\{x \in Q ;|\nabla u(x)|=1\}=P^{+} \cup P^{-} \text {. }
$$

To prove it, suppose first that $u\left(x^{0}\right)=\Phi\left(x^{0}\right), x^{0} \in Q$. If

$$
x^{0} \in R_{e} \cap\left\{d_{e}<C_{\mu}+d_{i}\right\} \quad \text { or } \quad x^{0} \in R_{i} \cap\left\{d_{e}>C_{\mu}+d_{i}\right\}
$$

then we can show, as in $\S 3$, that $\left|\nabla u\left(x^{0}\right)\right|=1$. On the other hand, $x^{0}$ cannot belong to $R_{0}^{+}$, for this would imply that $\left|\nabla u\left(x^{0}\right)\right|>1$.

Similarly, if $u\left(x^{0}\right)=\phi\left(x^{0}\right), x^{0} \in Q$, then $\left|\nabla u\left(x^{0}\right)\right|=1$.

Suppose next that $\phi(y)<u(y)<\Phi(y)$, for some $y \in Q$. We have to show that $|\nabla u(y)|<1$. If this is not true then, for some direction $l, \partial u(y) / \partial l=1$. Applying the maximum principle to $\partial u / \partial l$ in the component of $\{\phi<u<\Phi\}$ which contains $y$, we get a contradiction.

THEOREM 11.1. $R \subset E$, that is, the ridge is elastic.

The proof is similar to the proof of Theorem 3.2.

LEMMA 11.2. There exists a positive constant $c$ such that

$$
C_{\infty}-C_{\mu} \leqslant c / \mu \text { for all } \mu>0 .
$$

Proof. Set

$$
F_{\mu}(v)=\iint_{Q}|\nabla v|^{2}-\mu \iint_{Q^{*}} v
$$

Then $u$ minimizes $F_{\mu}(v)$ when $v$ varies in the convex set (1.6) (with $n=1$ ). Take

$$
v=\left\{\begin{array}{l}
\min \left\{d_{e}, C_{\infty}\right\} \text { in } Q, \\
C_{\infty} \text { in } Q_{1} .
\end{array}\right.
$$

Then $v \in K$ and (since $|\nabla u| \leqslant 1) u \leqslant v$ in $Q$. It follows that

$$
\iint_{Q^{*}} v-\iint_{Q^{*}} u \geqslant\left(C_{\infty}-C_{\mu}\right)\left(\text { meas } Q_{1}\right) .
$$

Since $|\nabla u| \leqslant 1,|\nabla v| \leqslant 1$, we obtain

$$
F_{\mu}(u)-F_{\mu}(v) \geqslant O(1)+\mu\left(C_{\infty}-C_{\mu}\right)\left(\text { meas } Q_{1}\right) .
$$

Recalling that $F_{\mu}(u)-F_{\mu}(v) \leqslant 0$, the assertion (11.7) follows. 
Definition. The set

$$
\begin{aligned}
R^{*}= & \left(R_{e} \cap\left\{d_{e}<C_{\infty}+d_{i}\right\}\right) \cup\left(R_{i} \cap\left\{d_{e}>C_{\infty}+d_{i}\right\}\right) \\
& \cup\left\{d_{e}=C_{\infty}+d_{i}\right\}
\end{aligned}
$$

is called the limit upper ridge. Let

$$
\delta^{*}(x)=\text { distance from } x \text { to } R^{*}, \quad x \in Q .
$$

THEOREM 11.3. There exists a positive constant $C$ such that

$$
Q \backslash P^{+} \subset\left\{x \in Q ; \delta^{*}(x)<C / \mu\right\}, \quad \mu>0 .
$$

Proof. Define $R_{\mu}^{*}$ and $\delta_{\mu}^{*}$ as $R^{*}, \delta^{*}$ when $C_{\infty}$ is replaced by $C_{\mu}$. Because of Lemma 11.2,

$$
\left|\delta_{\mu}^{*}(x)-\delta^{*}(x)\right| \leqslant c / \mu \quad(c \text { constant }) .
$$

The proof of Theorem 4.1 can be extended to the present case with $E$ replaced by $Q \backslash P^{+}$(since we only need the inequality $\Delta u \leqslant-\mu$ in the set where $u<\Phi)$ and with $\delta(x)$ replaced by $\delta_{\mu}^{*}(x)$. It follows that

$$
Q \backslash P^{+} \text {is contained in }\left\{x \in Q ; \delta_{\mu}^{*}(x)<C / \mu\right\} \text {. }
$$

Using (11.9), the assertion (11.8) follows.

We shall now improve Lemma 11.2.

THEOREM 11.4. There exists a positive constant $c$ such that

$$
C_{\infty}-C_{\mu} \leqslant c / \mu^{2} \text { for all } \mu>0 \text {. }
$$

Proof. We first claim that

$$
R^{*} \text { has finite length. }
$$

Indeed, consider first the set $R_{e}$. It is a finite union of sets of two kinds:

(a) the centers of curvatures of points varying on a $C^{3}$ arc;

(b) points not in (a) for which $d_{i}=d_{j}$, where $d_{i}, d_{j}$ are the distance functions to two $C^{3}$ arcs.

A set as in (a) lies on a $C^{1}$ curve and a set as in (b) is locally a $C^{2}$ curve (by the implicit function theorem). It follows that $R_{e}$ has a finite length. The proof of (11.11) is similar.

We now repeat the proof of Lemma 11.3 with $v$ the solution of the two obstacle problem with obstacles $\phi^{*}, \Phi^{*}$ corresponding to $\phi, \Phi$ when $C_{\mu}=$ $C_{\infty}$. By Theorem 11.3, the set where $u \neq \Phi$ is contained in a $(c / \mu)$-neighborhood of $R^{*}$ and, by (11.11), it then has measure $\leqslant c / \mu$. Similarly the set where $v \neq \Phi^{*}$ has measure $\leqslant c / \mu$. Since $|\nabla u|=|\nabla v|=1$ on the set where $u=\Phi, v=\Phi^{*}$, we conclude that

$$
\left.\left|\int_{Q}\right| \nabla u\right|^{2}-\int_{Q}|\nabla v|^{2} \mid \leqslant \frac{c}{\mu} .
$$


Proceeding now as in Lemma 11.2, the assertion (11.10) follows.

THEOREM 11.5. If $\partial Q_{1}$ is a convex polygon then

$$
P^{-} \backslash P^{+} \subset\left\{x \in Q ; \min _{1<i<l_{1}}\left|x-W_{i}\right|<\frac{1}{\mu}\right\}, \quad \mu>0,
$$

where $W_{i}$ are the vertices of $\partial Q_{1}$.

Proof. $\phi$ is piecewise linear or $C_{\mu}-r_{i}$, where $r_{i}(x)$ is the distance from $x$ to $W_{i}$, and $\phi \in C^{2}(Q)$. At each point $x^{0}$ where $u\left(x^{0}\right)=\phi\left(x^{0}\right)$ we must have $-\mu-\Delta \phi\left(x^{0}\right)>0$ (otherwise, the maximum principle applied to $u-\phi$ in a neighborhood of $x^{0}$ gives a contradiction). It follows that

$$
\mu \leqslant \max _{i} \frac{1}{r_{i}\left(x^{0}\right)}
$$

and (11.12) follows.

The proof of Theorem 11.5 extends to more general domains $Q_{1}$.

Let $V_{i}$ be a vertex of $\partial_{e} Q$ such that

$$
C_{\mu}<\operatorname{dist}\left(V_{i}, \partial Q_{1}\right)
$$

Then, in some $\bar{Q}$-neighborhood of $V_{i}$, the two-sided constraints on $u$ reduce to the single constraint $u<d_{e}$. Therefore, Theorems 5.1, 6.1 remain valid for such a vertex. Similarly, Theorems 7.1, 7.2 remain true provided

$$
C_{\mu}<\operatorname{dist}\left(\sigma_{0}, \partial Q_{1}\right)
$$

The methods of $\S 8$ can also be used for some special domains $Q$; see the example in the next section.

12. $u$ touches the lower obstacle. In this section we show that the set

$$
Q \cap\{u=\phi, u<\Phi\} \equiv P^{-} \backslash P^{+}
$$

is nonempty.

We shall denote by $\left|Q_{1}\right|$ the area of $Q_{1}$ and by $\left|\partial Q_{1}\right|$ the length of $\partial Q_{1}$.

TheOREM 12.1. Suppose $C_{\mu}<C_{\infty}$. Then the set (12.1) has positive measure provided $\mu>\left|Q_{1}\right| /\left|\partial Q_{1}\right|$.

Proof. Since $C_{\mu}<C_{\infty}$ we have $\phi<\Phi$ everywhere in $Q$. Thus it suffices to show that the set $u=\phi$, intersected with $Q$, has positive measure. We shall suppose that

$$
u>\phi \quad \text { a.e. in } Q
$$

and derive a contradiction. Let

$$
\zeta=\max \{0, \phi+\varepsilon-u\} .
$$

Since $C_{\mu}<C_{\infty}$, if $\varepsilon$ is sufficiently small then $u+\zeta$ belongs to the set

$$
K=\left\{v \in H_{0}^{1}\left(Q^{*}\right) ; \phi_{\varepsilon} \leqslant v \leqslant \Phi_{\varepsilon}\right\},
$$


where $\phi_{\varepsilon}, \Phi_{\varepsilon}$ are defined by (11.1), (11.2) with $C_{\mu}$ replaced by $C_{\mu}+\varepsilon$. Hence

$$
\iint_{Q} \nabla u \cdot \nabla \zeta-\mu \iint_{Q^{*}} \zeta \geqslant 0
$$

or

$$
-\iint_{Q} \Delta u \cdot \zeta+\int_{\partial Q_{1}} \frac{\partial u}{\partial \nu} \zeta-\mu \iint_{Q} \zeta-\mu \varepsilon\left|Q_{1}\right| \geqslant 0
$$

where $\nu$ is the normal pointing into $Q_{1}$. Since (12.2) holds, $\Delta u \geqslant-\mu$ a.e. in $Q$. It follows that

$$
\int_{\partial Q_{1}} \frac{\partial u}{\partial \nu} \varepsilon \geqslant \mu \varepsilon\left|Q_{1}\right|
$$

Noting that $|\partial u / \partial \nu| \leqslant 1$, we get $\left|\partial Q_{1}\right| \geqslant \mu\left|Q_{1}\right|$, thus contradicting our assumption on $\mu$.

Consider next the case where $C_{\mu}=C_{\infty}$. We shall assume that

there exists a vertex $W_{i}$ of $\partial Q_{1}$ such that $d_{e}\left(W_{i}\right)=\left|W_{i}-Z_{1}\right|$

$=\left|W_{i}-Z_{2}\right|=C_{\infty}\left(Z_{j} \in \partial_{e} Q\right) ;$ the rays $\gamma_{j}=\overrightarrow{W_{i} Z_{j}}$ from

$W_{i}$ to $Z_{j}$ form an angle $\theta, 0<\theta<\pi$; the sector obtained by

rotating $\overrightarrow{W_{i} Z}$ about $W_{i}$ from $Z_{1}$ to $Z_{2}$ (by an angle $\theta$ ) lies in $\bar{Q}$.

Denote by $\Sigma$ the domain bounded by $\gamma_{1}, \gamma_{2}$ and $\partial_{e} Q$.

THEOREM 12.2. If $C_{\mu}=C_{\infty}$ and if (12.3) holds then, for any neighborhood $N$ of $W_{i}, N \cap \Sigma$ intersects $\{u=\phi, u<\Phi\}$ in a set of positive measure.

Proof. Suppose the assertion is not true. Then, for some neighborhood $N$ of $W_{i}$,

$$
\Delta u \geqslant-\mu \text { a.e. in } N \cap \Sigma .
$$

Notice that $\Phi=\phi=u$ on $\gamma_{1} \cup \gamma_{2}$ and this function is linearly decreasing along each $\gamma_{i}$, with slope -1 . We shall compare $u$ with the function $v$ :

$$
\begin{aligned}
\Delta v & =-\mu \quad \text { in } N, \\
v & =u \text { on } \partial \Sigma .
\end{aligned}
$$

In view of (12.4), $v \geqslant u$ in $\Sigma$.

Let $l$ be the linear function satisfying $l=u$ on $\gamma_{1} \cup \gamma_{2}$. Then $|\nabla l|>1$ (since $\theta<\pi$ ). The function

$$
\tilde{v}=v+\mu r_{i}^{2} / 4-l
$$

is in $C^{1+\varepsilon}(\varepsilon>0)$ in $N \cap \Sigma$ (as seen by using the conformal mapping $z=g(\tilde{z})$ as in the proof of Theorem 6.1). It follows that

$$
\partial v / \partial \gamma<-1 \text { at } W_{i}
$$


where $\gamma$ is the bisector of $\gamma_{1}, \gamma_{2}$. Consequently,

$$
\partial u / \partial \gamma<-1 \text { at } W_{i},
$$

which is impossible since $|\nabla u| \leqslant 1$.

EXAMPLE. Let $\partial_{e} Q$ and $\partial Q_{1}$ be concentric squares with parallel sides of lengths $2 b$ and $2 a$, respectively. If $C_{\mu}<C_{\infty}$ then, by Theorem 12.1, the set $P^{-} \backslash P^{+}$has positive measure provided $\mu>a / 2$. By Theorem 11.4, this set lies in a $(1 / \mu)$-neighborhood of the vertices of $\partial Q_{1}$.

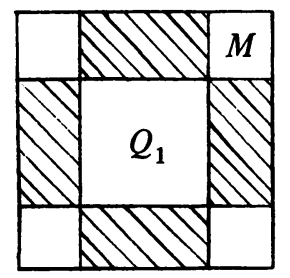

If $C_{\mu}=C_{\infty}$ then the four shaded squares in the accompanying figure are plastic with $u=\phi=\Phi$. To study the shape of the elastic set in one of the four remaining squares of $Q$, say $M$, we choose the coordinate system so that

$$
M=\{(x, y) ; 0<x<h, 0<y<h\}, \quad h=b-a .
$$

Let $\pi_{1}=\{(x, 0) ; 0<x<h\}, \pi_{2}=\{(h, y) ; 0<y<h\}, \pi=\{(x, x) ; 0<x$ $<h\}$ and denote by $M_{1}$ the triangle with sides $\pi_{1}, \pi_{2}, \pi$. On $\pi_{1}, u_{x}=-1$ and therefore $u_{\theta}=u_{y}=0$ on $\pi_{1}$. Next, $u_{\theta}=0$ on $\pi$ by symmetry. Clearly also $u_{\theta} \geqslant 0$ on $\pi_{2}$. Since, in $M_{1}$,

$$
\Phi(x, y)=h-x, \quad \phi(x, y)=b-a-r,
$$

we have on the free boundary:

$$
\begin{array}{ll}
u_{\theta}=r_{\theta}=0 & \text { where } u=\phi, \\
y_{\theta}=\Phi_{\theta}=r \sin \theta \geqslant 0 & \text { where } u=\Phi .
\end{array}
$$

Applying the maximum principle to $u_{\theta}$ in $E \cap M_{1}$ we deduce that $u_{\theta}>0$. Similarly $u_{y} \leqslant 0$ in $E \cap M$. It follows that

$$
(u-\phi)_{\theta} \geqslant 0, \quad(u-\Phi)_{y} \leqslant 0 \text { in } E \cap M_{1} .
$$

Observe that the sets where $u=\phi, u=\Phi$ do not intersect in $M_{1}$ (since $\phi<\Phi$ in $M_{1}$ ). From this fact and from (12.5) we deduce that the free boundary in $M_{1}$ consists of two curves:

$$
\begin{array}{ll}
\Gamma_{1}: r=\rho(\theta), & 0<\theta<\pi / 4, \\
\Gamma_{2}: y=k(x), & x_{0}<x<1,
\end{array}
$$

where $\rho(\theta)$ is decreasing and $k(x)$ is increasing. Further (by the proof of Theorem 8.3) $\Gamma_{1}$ and $\Gamma_{2}$ are continuous up to the boundary and their end-points on $y=0$ coincide. By Theorem 12.2 the curve $\Gamma_{1}$ is not the trivial 
curve $\rho(\theta)=0$, i.e., $\rho(\theta)>0$ if $0<\theta<\pi / 4$. The curve $\Gamma_{2}$ may reduce to one point (when $x_{0}=0$ ).

Some numerical computations for $Q$ as in the above example have been carried out by F. S. Shaw and reported by Southwell [12]. Numerical work based on the assumption that (12.1) is the empty set was carried out in [10], but some of the pictures given in that work regarding the elastic and plastic regions do not conform to those given here and in [12].

\section{REFERENCES}

1. H. Brézis and M. Sibony, Équivalence de deux inéquations variationnelles et applications, Arch. Rational Mech. Anal. 41 (1971), 254-265.

2. H. Brézis and G. Stampacchia, Sur la régularité de la solution d'inéquations elliptiques, Bull. Soc. Math. France 96 (1968), 153-180.

3. Rational Mech. Anal. 61 (1976), 1-18.

4. L. A. Caffarelli and A. Friedman, The dam problem with two layers, Arch. Rational Mech. Anal. 68 (1978), 125-154.

5. L. A. Caffarelli and N. M. Riviere, Smoothness and analyticity of free boundaries in variational inequalities, Ann. Scuola Norm. Sup. Pisa 3 (1976), 289-310.

6. The smoothness of the elastic-plastic free boundary of a twisted bar, Proc. Amer. Math. Soc. 63 (1977), 56-58.

7. On the Lipschitz character of the stress tensor when twisting an elastic plastic bar, Arch. Rational Mech. Anal. (to appear).

8. A. Friedman and R. Jensen, Convexity of the free boundary in the Stefan problem and in the dam problem, Arch. Rational Mech. Anal. 67 (1977), 1-24.

9. C. Gerhardt, Regularity of solutions of nonlinear variational inequalities with a gradient bound as constraint, Arch. Rational Mech. Anal. 58 (1975), 309-315.

10. R. Glowinski and H. Lanchon, Torsion élastoplastique d'une barre cylindrique de section multiconvexe, J. Méchanique 12 (1973), 151-171.

11. H. Lanchon, Torsion élastoplastique d'un barre cylindrique de section simplement ou multiplement convexe, J. Méchanique 13 (1974), 267-320.

12. R. V. Southwell, On the computation of strain and displacement in a prism plastically strained by torsion, Quart. J. Mech. Appl. Math. 2 (1949), 385-397.

13. T. W. Ting, Elastic-plastic torsion of square bar, Trans. Amer. Math. Soc. 123 (1966), $369-401$.

14. The ridge of a Jordan domain and completely plastic torsion, J. Math. Mech. 15 (1966), 15-47.

15. __ Elastic-plastic torsion problem. II, Arch. Rational Mech. Anal. 25 (1967), 342-365.

16. __ Elastic-plastic torsion problem. III, Arch. Rational Mech. Anal. 34 (1969), $228-244$.

17. __ Elastic-plastic torsion of convex cylindrical bars, J. Math. Mech. 19 (1969), 531-551.

18. _ Elastic-plastic torsion of simply connected cylindrical bars, Indiana Univ. Math. J. 20 (1971), 1047-1076.

19. Elastic-plastic torsion problem over multiply connected domains, Ann. Scuola Norm. Sup. Pisa. 4 (4) (1977), 291-312.

School of Mathematics, University of Minnesota, Minneapolis, Minnesota 55455

DePartment of Mathematics, NorthWestern University, Evanston, Illinois 60201 